\title{
Two-photon coherent states of the radiation field*
}

\author{
Horace P. Yuen \\ Research Laboratory of Electronics, Massachusetts Institute of Technology, Cambridge, Massachusetts 02139
}

(Received 26 June 1975; revised manuscript received 3 March 1976)

\begin{abstract}
The concept of a two-photon coherent state is introduced for applications in quantum optics. It is a simple generalization of the well-known minimum-uncertainty wave packets. The detailed properties of two-photon coherent states are developed and distinguished from ordinary coherent states. These two-photon coherent states are mathematically generated from coherent states through unitary operators associated with quadratic Hamiltonians. Physically they are the radiation states of ideal two-photon lasers operating far above threshold, according to the self-consistent-field approximation. The mean-square quantum noise behavior of these states, which is basically the same as those of minimum-uncertainty states, leads to applications not obtainable from coherent states or one-photon lasers. The essential behavior of two-photon coherent states is unchanged by small losses in the system. The counting rates or distributions these states generate in photocount experiments also reveal their difference from coherent states.
\end{abstract}

\section{INTRODUCTION}

In a recent study ${ }^{1}$ of quantum communication theory it was found that if optical radiations can be generated in a minimum-uncertainty state $^{2,3}$ rather than a coherent state, ${ }^{4}$ significant performance improvement may result in an optical communication system. For a fixed radiation mode of frequency $\omega$ with photon annihilation operator $a$, the coherent states $|\alpha\rangle(a|\alpha\rangle=\alpha|\alpha\rangle)$ have relatively large quantum fluctuations when $\omega / 2 \pi \geqslant 10^{12} \mathrm{~Hz}$. Writing $a=a_{1}+i a_{2}$ for self-adjoint $a_{1}, a_{2}$, a coherent state $|\alpha\rangle$ gives $\left\langle\Delta a_{1}^{2}\right\rangle=\left\langle\Delta a_{2}^{2}\right\rangle=\frac{1}{4}$. A noise energy ${ }^{5}$ $\frac{1}{4} \hbar \omega$ is then obtained if either $a_{1}$ or $a_{2}$ is measured in optical homodyne detection. This quantum noise is frequently dominant over other noise sources in optical communications; for example, an equivalent noise temperature of $\simeq 3400^{\circ} \mathrm{K}$ is obtained for $\frac{1}{4} \hbar \omega$ at the YAlG laser frequency. However, states with $\left\langle\Delta a_{1}^{2}\right\rangle \ll \frac{1}{4}$ and correspondingly larger $\left\langle\Delta a_{2}^{2}\right\rangle$ are permitted by the uncertainty principle $\left\langle\Delta a_{1}^{2}\right\rangle\left\langle\Delta a_{2}^{2}\right\rangle \geqslant \frac{1}{16}$, such as the minimum uncertainty states with $\left\langle\Delta a_{1}^{2}\right\rangle\left\langle\Delta a_{2}^{2}\right\rangle=\frac{1}{16}$. If one can generate such small $\left\langle\Delta a_{1}^{2}\right\rangle$ states by an explicit physical process, it should not be surprising that they may be profitably used in many applications.

Minimum-uncertainty states cannot be obtained, however, from available optical sources, all of which generate coherent states and their random or classical superpositions. An investigation of the possible ways to generate minimum-uncertainty states leads to a broad class of radiation states that we call "two-photon coherent states" (TCS's). They include the minimum-uncertainty states of which the coherent state is a special case. A detailed study of these TCS's is reported in this article. A few of our results have been briefly stated in Ref. 6 .
In general, a two-photon coherent state differs from a coherent state in several ways: they are generated by different photon processes, they have different quantum statistical properties, and they have different coherence properties. Basically, coherent states are generated from ideal onephoton stimulated processes, whereas TCS's are obtained from ideal stimulated two-photon processes for two photons of the same mode. The usual multiphoton parametric processes ${ }^{7}$ are one-photon processes in the present sense, as they involve a single photon from each separate mode. The quantum noise properties of TCS's are basically the same as those of minimum-uncertainty states.

Minimum-uncertainty states and coherent states can be easily confused. The above illustration in terms of $\left\langle\Delta a_{1}^{2}\right\rangle$ and $\left\langle\Delta a_{2}^{2}\right\rangle$ is a good indication of their difference, if we note that they describe the fluctuations of the field variables directly measured in a homodyne experiment. Further careful discussions will be found in Sec. III E and elsewhere in the paper.

Minimum-uncertainty states were studied at the very early days of quantum mechanics; the Kennard packets ${ }^{8}$ are particularly familiar in the context of electrons. It is therefore no surprise that much previous work has touched on various aspects relating to the present two-photon coherent states, including much work on quantum field theory and interacting Bose fluids. It is impossible for the present author to give an accurate historical survey of the literature pertaining to minimumuncertainty states and their generation by quadratic Hamiltonians. The particularly relevant papers now known to the author include Refs. 9-13 in quantum optics and Refs. 14-17 in quantum field theory. Nevertheless, it appears that TCS's have not been systematically studied before, at least in 
the context of quantum optics. These and other additional papers will be mentioned in relation to some of our results whenever appropriate.

This work is not at all a study of possible mathematical generalizations of coherent states. In fact, the TCS's can be considered as "coherent states" in a broad sense, as discussed later. However, they should be distinguished from the coherent states of Glauber, ${ }^{4}$ because in quantum optics the term coherent state is usually understood in the Glauber sense which carries the implication of equal fluctuations in the quadratures $a_{1}$ and $a_{2}$.

In Sec. II we establish notations and briefly recapitulate the basic results in quantum optics used in this paper. For a single degree of freedom, the definition and properties of two-photon coherent states are developed in Sec. III, together with a discussion of the contrast between TCS's and ordinary coherent states. The generation of TCS's from quadratic Hamiltonians is presented in Sec. IV, with illustrative examples. The general structure and properties of the corresponding unitary transformation are fully determined. Physical interpretation of quadratic Hamiltonians is developed in Sec. V in terms of stimulated two-photon processes, which include two-photon lasers and degenerate parametric amplifiers. We argue that TCS's describe ideal two-photon lasing states in analogy to the coherent-state description of onephoton lasers. The approximation which leads to this conclusion is formalized in the Appendix. We also show that the mean-square noise behavior of TCS's remains essentially the same when the effect of small damping and additive noise are included. In Sec. VI we briefly discuss the coherence properties and counting statistics of two-photon coherent states. The usefulness of TCS's in certain applications is brought out in Sec. VII. The detailed theory of multimode TCS's will be given in a separate treatment.

\section{COHERENT STATES AND OPERATOR REPRESENTATION}

For a single mode of the field with photon annihilation operator $a$, a coherent state ${ }^{18,19}|\alpha\rangle$ is an eigenstate of $a$,

$$
a|\alpha\rangle=\alpha|\alpha\rangle \text {. }
$$

It can be obtained from the vacuum state $|0\rangle$ via the unitary displacement operator $D(\alpha) \equiv e^{\alpha a^{\dagger}-\alpha^{*} a}$,

$$
|\alpha\rangle=D(\alpha)|0\rangle \text {. }
$$

From (2.2),

$$
\langle n \mid \alpha\rangle=\alpha^{n}(n !)^{-1 / 2} e^{-|\alpha|^{2 / 2}} .
$$

If the mode has a harmonic time variation with frequency $\omega$, we have the usual canonical variables $(q, p)$,

$$
q \equiv(\hbar / 2 \omega)^{1 / 2}\left(a^{\dagger}+a\right), \quad p \equiv i\left(\frac{1}{2} \hbar \omega\right)^{1 / 2}\left(a^{\dagger}-a\right) .
$$

In general, we can introduce two dimensionless self-adjoint operators $\left(a_{1}, a_{2}\right)$,

$$
a \equiv a_{1}+i a_{2} ; \quad a_{1}=a_{1}^{\dagger}, \quad a_{2}=a_{2}^{\dagger} .
$$

We also write

$$
\alpha \equiv \alpha_{1}+i \alpha_{2} ; \quad \alpha_{1}, \alpha_{2} \text { real, }
$$

and refer to $\left(a_{1}, a_{2}\right)$ or $\left(\alpha_{1}, \alpha_{2}\right)$ as the quadrature components (quantum or classical) of the radiation mode. These quadrature components are the natural variables in describing a TCS, even when the mode has a single frequency. Defining the eigenstates $\left|\alpha_{1}\right\rangle_{1}$ of $a_{1},\left|\alpha_{2}\right\rangle_{2}$ of $a_{2}$,

$$
a_{1}\left|\alpha_{1}\right\rangle_{1}=\alpha_{1}\left|\alpha_{1}\right\rangle_{1}, \quad a_{2}\left|\alpha_{2}\right\rangle_{2}=\alpha_{2}\left|\alpha_{2}\right\rangle_{2},
$$

we have

$$
{ }_{1}\left\langle\alpha_{1} \mid \alpha^{\prime}\right\rangle=(2 / \pi)^{1 / 4} \exp \left[-\left(\alpha_{1}-\alpha^{\prime}\right)^{2}+\frac{1}{2} \alpha^{\prime}\left(\alpha^{\prime}-\alpha^{\prime *}\right)\right] .
$$

From (2.6) it follows easily that

$$
\begin{aligned}
& \langle a\rangle=\alpha,\langle N\rangle=|\alpha|^{2}, \\
& \left\langle\Delta a_{1}^{2}\right\rangle=\left\langle\Delta a_{2}^{2}\right\rangle=\frac{1}{4},
\end{aligned}
$$

where $\left\langle\Delta M^{2}\right\rangle \equiv\left\langle(M-\langle M\rangle)^{2}\right\rangle$ for an operator $M$. The angular bracket $\langle M\rangle$ represents the expected value $\operatorname{tr} \rho M$ of $M$ with respect to a quantum state $\rho$ which may be either pure or mixed. The uncertainty principle on $\left(a_{1}, a_{2}\right)$ is

$$
\left\langle\Delta a_{1}^{2}\right\rangle\left\langle\Delta a_{2}^{2}\right\rangle \geqslant \frac{1}{16} .
$$

Thus $|\alpha\rangle$ is a minimum-uncertainty state. From the arithmetic-geometric mean inequality, (2.11) implies

$$
\left\langle\Delta a_{1}^{2}\right\rangle+\left\langle\Delta a_{2}^{2}\right\rangle \geqslant \frac{1}{2},
$$

so that $|\alpha\rangle$ can be characterized as the states with both minimum-uncertainty product and minimumuncertainty sum.

The differential operator representation of $M\left(a^{\dagger}, a\right)$ in the coherent-state representation is ${ }^{20,19}$

$$
\left\langle\alpha\left|M\left(a^{\dagger}, a\right)\right| \psi\right\rangle=M\left(\alpha^{*}, \frac{\alpha}{2}+\frac{\partial}{\partial \alpha^{*}}\right)\langle\alpha \mid \psi\rangle,
$$

from which one can show

$$
\left\langle\alpha\left|M\left(a^{\dagger}, a\right)\right| \beta\right\rangle=\langle\alpha \mid \beta\rangle M^{(n)}\left(\alpha^{*}, \beta\right),
$$

where

$$
M^{(n)}\left(\alpha^{*}, \alpha\right) \equiv\langle\alpha|M| \alpha\rangle
$$

is the normal-order for $\mathrm{m}^{3}$ of $M$. Note that it is important to keep the separate dependence of $M^{(n)}$ 
on $\alpha^{*}$ and $\alpha$ in $(2.14)-M^{(n)}$ is a function of two complex variables $\left(\alpha^{*}, \alpha\right)$. Equation ( 2.14$)$ explicitly shows the fact ${ }^{19}$ that $M^{(n)}\left(\alpha^{*}, \alpha\right)$ uniquely determines $M$. In terms of $U^{(n)}\left(\alpha^{*}, \alpha ; t, t_{c}\right)$, the Schrödinger equation $i \hbar \partial U / \partial t=H\left(a^{\dagger}, a\right) U$ becomes $^{19}$

$$
i \hbar \frac{\partial U^{(n)}}{\partial t}=H\left(\alpha^{*}, \alpha+\frac{\partial}{\partial \alpha^{*}}\right) U^{(n)} .
$$

These results are at least valid for bounded operators or unbounded operators which are polynomials in $a$ and $a^{\dagger}$.

In a number of mathematical senses, ${ }^{21-24}$ any density operator $\rho$ can be expressed in the diagonal $P$ representation, ${ }^{4,25}$

$\rho=\int P\left(\alpha^{*}, \alpha\right)|\alpha\rangle\langle\alpha| d^{2} \alpha, d^{2} \alpha \equiv d(\operatorname{Re} \alpha) d(\operatorname{Im} \alpha)$.

Let $\chi_{N}\left(\eta^{*}, \eta\right)$ be the normally ordered characteristic function

$$
\chi_{N}\left(\eta^{*}, \eta\right) \equiv \operatorname{tr}\left(\rho e^{\eta a^{\dagger}} e^{-\eta^{*} a}\right),
$$

then $P\left(\alpha^{*}, \alpha\right)$ is the two-dimensional Fourier transform of $\chi_{N}\left(\eta^{*}, \eta\right),{ }^{26,21}$

$$
P\left(\alpha^{*}, \alpha\right)=\int e^{\eta^{*} \alpha-\alpha^{*} \eta} \chi_{N}\left(\eta^{*}, \eta\right) d^{2} \eta / \pi^{2} .
$$

It has not been demonstrated that $P\left(\alpha^{*}, \alpha\right)$ provides a convenient tool in actual calculation when it is not sufficiently well behaved.

Being the trace of the product of a bounded operator and a trace-class operator, the characteristic functions $\chi_{N}\left(\eta^{*}, \eta\right), \chi_{A}\left(\eta^{*}, \eta\right)$, and $\chi_{W}\left(\eta^{*}, \eta\right)$ are always well defined,

$$
\begin{aligned}
& \chi_{A}\left(\eta^{*}, \eta\right) \equiv \operatorname{tr}\left(\rho e^{-\eta^{*} a} e^{\eta a^{\dagger}}\right), \\
& \chi_{W}\left(\eta^{*}, \eta\right) \equiv \operatorname{tr}\left(\rho e^{-\eta^{*} a+\eta a^{\dagger}}\right) .
\end{aligned}
$$

The Fourier transform of $\chi_{W}\left(\eta^{*}, \eta\right)$ is the Wigner distribution and that of $\chi_{A}\left(\eta^{*}, \eta\right)$ is $\rho^{(n)}\left(\alpha^{*}, \alpha\right),{ }^{27,21}$ $\rho^{(n)}\left(\alpha^{*}, \alpha\right) \equiv\langle\alpha|\rho| \alpha\rangle$,

$$
\begin{aligned}
& W\left(\alpha^{*}, \alpha\right)=\int \chi_{W}\left(\eta^{*}, \eta\right) e^{\eta^{*} \alpha-\eta \alpha^{*}} d^{2} \eta / \pi^{2}, \\
& \chi_{A}\left(\eta^{*}, \eta\right)=\int \rho^{(n)}\left(\alpha^{*}, \alpha\right) e^{\eta \alpha^{*}-\alpha \eta^{*}} d^{2} \alpha / \pi .
\end{aligned}
$$

The characteristic functions are related by

$$
\chi_{\boldsymbol{A}}\left(\eta^{*}, \eta\right)=e^{-|\eta|^{2}} \chi_{N}\left(\eta^{*}, \eta\right)=e^{-|\eta|^{2} / 2} \chi_{W}\left(\eta^{*}, \eta\right),
$$

which also implies relations between $P\left(\alpha^{*}, \alpha\right)$, $\rho^{(n)}\left(\alpha^{*}, \alpha\right)$, and $W\left(\alpha^{*}, \alpha\right)$.

The function $\rho^{(n)}\left(\alpha^{*}, \alpha\right)$ is always well behaved, in contrast to $P\left(\alpha^{*}, \alpha\right)$, and is also quite convenient for moment calculations. Furthermore, it specifies $\rho$ uniquely as in (2.14) and is the prob- ability density function ${ }^{28}$ describing the outcome statistics of a simultaneous quantum measurement of two noncommuting observables ${ }^{29,} 30$ (or a measurement of the non-self-adjoint operator $a$, optical heterodyning). ${ }^{31}$ We will use $\rho^{(n)}\left(\alpha^{*}, \alpha\right)$ or the characteristic functions to represent a density operator, particularly because $P\left(\alpha^{*}, \alpha\right)$ for a TCS is too singular. In terms of $\rho^{(n)}$, we have

$$
\begin{aligned}
& \rho=\int e^{\eta \alpha^{*}-\alpha \eta \eta^{*}} \rho^{(n)}\left(\alpha^{*}, \alpha\right) e^{-\eta a^{\dagger}} e^{\eta^{*} a} d^{2} \alpha d^{2} \eta / \pi^{2}, \\
& \operatorname{tr} \rho M=\int\left[\chi_{A}^{M}\left(\eta^{*}, \eta\right)\right]{ }^{*} \chi_{A}^{\rho}\left(\eta^{*}, \eta\right) e^{|\eta|^{2}} d^{2} \eta / \pi, \\
& \text { if } \chi_{A}^{M}\left(\eta^{*}, \eta\right)=\operatorname{tr}\left(M e^{-\eta^{*} a} e^{\eta a^{\dagger}}\right) \text { exists. }
\end{aligned}
$$

\section{TWO-PHOTON COHERENT STATES}

\section{A. Definition}

For a fixed radiation mode with photon annihilation operator $a$, let

$$
b \equiv \mu a+\nu a^{\dagger}
$$

for a pair of $c$ numbers $\mu, \nu$ obeying

$$
|\mu|^{2}-|\nu|^{2}=1 \text {. }
$$

It follows from (3.2) that

$$
\left[b, b^{\dagger}\right]=1 \text {. }
$$

Any transformation $b\left(a^{\dagger}, a\right)$ which leaves the commutator invariant as in (3.3) is called a canonical transformation. The change of variables from $\left(a, a^{\dagger}\right)$ to $\left(b, b^{\dagger}\right)$ according to (3.1) and (3.2) is therefore a linear canonical transformation. A theorem of Von Neumann ${ }^{32,17}$ asserts that every canonical transformation can be represented as a unitary transformation, i.e.,

$$
b\left(a^{\dagger}, a\right)=U a U^{\dagger}
$$

for a unitary operator $U$. The structure and physical realization of the $U_{L}$ that leads to the linear canonical transformation (3.1) are discussed in Secs. IV and V;

$$
U_{L} a U_{L}^{\dagger} \equiv \mu a+\nu a^{\dagger} .
$$

The commutator (3.3) or Eq. (3.4) provides $b$ with properties exactly similar to those of $a$. One obtains the following in a way identical to the usual derivation for $N$ and $a:{ }^{3}$ Let $N_{g}$ be the "quasiphoton" number operator,

$$
N_{g} \equiv b^{\dagger} b=U_{L} N U_{L}^{\dagger} \text {. }
$$

Then $N_{g}$ has discrete positive eigenvalues $n_{g}$ with ground state $\left|0_{g}\right\rangle$,

$$
\begin{aligned}
& N_{g}\left|m_{g}\right\rangle=m_{g}\left|m_{g}\right\rangle, \quad N_{g}\left|0_{g}\right\rangle=0, \\
& \left|m_{g}\right\rangle=U_{L}|m\rangle .
\end{aligned}
$$


Similar to $|n\rangle$, the states $\left|m_{g}\right\rangle$ can be expressed in the form

$$
\left|m_{g}\right\rangle=\left(b^{\dagger}\right)^{m}(m !)^{-1 / 2}\left|0_{g}\right\rangle \text {. }
$$

They are complete orthonormal. Furthermore, $b$ acts as the lowering operator for $\left|m_{g}\right\rangle$. The quasiparticle interpretation of $b$ and $\left|m_{g}\right\rangle$ is discussed in Sec. IV A.

The TCS's $|\beta\rangle_{g}$ are defined to be the eigenstates of $b$ with eigenvalues $\beta$,

$$
b|\beta\rangle_{g}=\beta|\beta\rangle_{g} .
$$

The $\mu, \nu$ dependence of a TCS has been suppressed in the notation $|\beta\rangle_{g}$. When necessary, we will use the more complete notation $|\beta ; \mu, \nu\rangle$. With $\nu=0$, $|\beta\rangle_{g}$ become the ordinary coherent states. In our later references to a TCS, it should be implicitly understood that $\nu \neq 0$.

From (3.4), there is an $U_{L}$ independent of $\beta$ which gives

$$
|\beta\rangle_{g}=U_{L}|\beta\rangle
$$

for each state $|\beta\rangle$. Therefore in parallel with $|\beta\rangle$

$$
\begin{aligned}
& |\beta\rangle_{g}=D_{g}(\beta)|0\rangle_{g}, \quad D_{g}(\beta) \equiv e^{\beta b^{\dagger}-\beta^{*} b}, \\
& \int|\beta\rangle_{g}\langle\beta| d^{2} \beta / \pi=I, \\
& { }_{g}\left\langle\beta \mid \beta^{\prime}\right\rangle_{g}=\exp \left(\beta^{*} \beta^{\prime}-\frac{1}{2}|\beta|^{2}-\frac{1}{2}\left|\beta^{\prime}\right|^{2}\right) .
\end{aligned}
$$

If the notation $|\beta ; \mu, \nu\rangle$ is not used, the same $\mu, \nu$ is understood to apply to all of the $|\beta\rangle_{g}$ in an equation.

From (3.13), any state vector $|\psi\rangle$ can be expanded in terms of $|\beta\rangle_{g}$. Similarly, diagonal operator representation can be developed,

$$
M=\int M^{(a)}\left(\beta^{*}, \beta\right)|\beta\rangle_{g}\langle\beta| d^{2} \beta / \pi,
$$

for the antinormal order form $M^{(a)}\left(\beta^{*}, \beta\right)$ of $M$. In the $|\beta\rangle_{g}$ representation, we have [similar to $(2.21)$ ]

$$
{ }_{g}\left\langle\beta\left|M\left(b^{\dagger}, b\right)\right| \psi\right\rangle=M\left(\beta^{*}, \frac{\beta}{2}+\frac{\partial}{\partial \beta^{*}}\right){ }_{g}\langle\beta \mid \psi\rangle .
$$

Other properties associated with $|\alpha\rangle$ carry over identically to $|\beta\rangle_{g}$.

The results of this subsection on $|\beta\rangle_{g}$ and $\left|m_{g}\right\rangle$ depends only on the relation (3.4), and not on the more specific (3.5). The specific characteristics of (3.1) are developed below. Certain properties of TCS's have been discussed by Stoler ${ }^{9}$ in the context of minimum-uncertainty states.

\section{B. Wave functions}

The general coherent-state wave function $\langle\alpha \mid \beta\rangle_{g}$ of a TCS is first determined as follows: From (3.1), (3.10), and (2.13) we obtain

$$
\frac{\partial}{\partial \alpha^{*}}\langle\alpha \mid \beta\rangle_{g}=\left(\frac{\beta}{\mu}-\frac{\alpha}{2}-\frac{\nu \alpha^{*}}{\mu}\right)\langle\alpha \mid \beta\rangle_{g} .
$$

The solution of (3.17) is of the form

$$
\langle\alpha \mid \beta\rangle_{g}=\exp \left[\alpha * \beta / \mu-\frac{1}{2}|\alpha|^{2}-\nu \alpha^{*^{2}} / 2 \mu+f\left(\beta^{*}, \beta\right)\right] .
$$

The $\left(\alpha^{*}, \alpha\right)$-independent $f$ can be determined from (3.16), (3.1), (2.13), and

$$
\begin{aligned}
{ }_{g}\langle\beta|b| \alpha\rangle & =\left(\frac{\beta}{2}+\frac{\partial}{\partial \beta^{*}}\right)_{g}\langle\beta \mid \alpha\rangle \\
& =\left(\mu \alpha+\frac{\nu \alpha^{*}}{2}+\nu \frac{\partial}{\partial \alpha}\right)_{g}\langle\beta \mid \alpha\rangle,
\end{aligned}
$$

so that the $\beta$-dependent part of $f$ in (3.18) is $-\frac{1}{2}|\beta|^{2}$ $+\nu^{*} \beta^{2} / 2 \mu$. From the normalization

$$
\int\left|\langle\alpha \mid \beta\rangle_{g}\right|^{2} d^{2} \alpha / \pi=1
$$

$\langle\alpha \mid \beta\rangle_{g}$ is thus determined up to a $\left(\alpha^{*}, \alpha ; \beta^{*}, \beta\right)$ independent phase in the form

$$
\begin{aligned}
\langle\alpha \mid \beta\rangle_{g}=\mu^{-1 / 2} \exp [ & -\frac{1}{2}|\alpha|^{2}-\frac{1}{2}|\beta|^{2}-(\nu / 2 \mu) \alpha^{* 2} \\
& \left.+\left(\nu^{*} / 2 \mu\right) \beta^{2}+(1 / \mu) \alpha^{*} \beta+i \theta_{0}\right]
\end{aligned}
$$

for a real $\theta_{0}$. The normalization constant is calculated from the normalization of Gaussian integrals. $^{20}$ For $\mu=1, \nu=0,(3.20)$ reduces to the usual form of $\langle\alpha \mid \beta\rangle$ for $\theta_{0}=0$, a choice of phase we will adopt for $\langle\alpha \mid \beta\rangle_{g}$.

While we have

$$
\left\langle m_{\boldsymbol{g}} \mid \beta\right\rangle_{\boldsymbol{g}}=\beta^{m}(m !)^{-1 / 2} e^{-|\beta|^{2} / 2},
$$

similar to (2.3), the function $\langle m \mid \beta\rangle_{g}$ takes a more complicated form which can be determined as follows: As a function of the complex variables $z$ and $t, e^{2 z t-t^{2}}$ can be expanded in the Taylor series

$$
e^{2 z t-t^{2}}=\sum_{n=0}^{\infty} \frac{H_{n}(z) t^{n}}{n !}, \quad|t|<\infty,
$$

where $H_{n}(z)$ is the $n$th Hermite polynomial with complex argument $z$. Writing

$$
\langle\alpha \mid \beta\rangle_{g}=\sum_{n}\langle\alpha \mid n\rangle\langle n \mid \beta\rangle_{g}
$$

and using (3.20) and (2.3), we find

$$
\begin{aligned}
\langle n \mid \beta\rangle_{g}= & (n ! \mu)^{-1 / 2}(\nu / 2 \mu)^{n / 2} H_{n}\left[\beta(2 \mu \nu)^{-1 / 2}\right] \\
& \times \exp \left[-\frac{1}{2}|\beta|^{2}+\left(\nu^{*} / 2 \mu\right) \beta^{2}\right] .
\end{aligned}
$$

For $\mu=1, v=0$, the asymptotic forms of $H_{n}(z)$ for various ${ }^{33}$ argz all lead to (2.3), as they must. Equation (3.23) shows that the counting statistics of a TCS is far from the Poisson (2.3). 
The wave function ${ }_{1}\left\langle\alpha_{1} \mid \beta\right\rangle_{g}$ can be readily determined from (3.20) and (2.8). Thus we find

$$
{ }_{1}\left\langle\alpha_{1} \mid \beta\right\rangle_{g}=\left(\frac{2}{\pi}\right)^{1 / 4}(\mu-\nu)^{-1 / 2} \exp \left(-\frac{1}{2}\left|\hat{k}^{\prime}\right|^{2}-\frac{\mu^{*}-\nu^{*}}{2(\mu-\nu)} \beta^{2}+2 \alpha_{1} \frac{\beta}{\mu-\nu}-\frac{\mu+\nu}{\mu-\nu} \alpha_{1}^{2}\right) \text {. }
$$

From (3.20) we have

$$
\begin{aligned}
\left\langle\beta ; \mu, \nu \mid \beta_{0} ; \mu_{0}, \nu_{0}\right\rangle= & \left(\mu_{0} \mu^{*}-\nu_{0} \nu^{*}\right)^{-1 / 2} \\
& \times \exp \left(-\frac{1}{2}|\beta|^{2}-\frac{1}{2}\left|\beta_{0}\right|^{2}+\frac{1}{\mu_{0} \mu^{*}-\nu_{0} \nu^{*}} \beta^{*} \beta_{0}-\frac{\nu_{0} \mu-\mu_{0} \nu}{2\left(\mu_{0} \mu^{*}-\nu_{0} \nu^{*}\right)} \beta^{* 2}+\frac{\left(\nu_{0} \mu-\mu_{0} \nu\right)^{*}}{2\left(\mu_{0} \mu^{*}-\nu_{0} \nu^{*}\right)} \beta_{0}^{2}\right) .
\end{aligned}
$$

Note the Gaussian form of (3.24) and (3.25). Various reduction of (3.24) and (3.25) to the previous formulas can be easily observed. The relative phases of (3.20), (3.21), (3.24), and (3.25) are mutually consistent, as well as being consistent with the above wave functions for the coherent states.

From

$$
\langle n \mid \beta\rangle_{g}=\sum_{m}\left\langle n \mid m_{g}\right\rangle\left\langle m_{g} \mid \beta\right\rangle_{g}
$$

for the quasiphoton number states $\left|m_{\boldsymbol{g}}\right\rangle$ we obtain $\left\langle n \mid m_{g}\right\rangle=Q_{1}(\mu, \nu ; m, n)$,

$m \leqslant n, \quad m$ odd, $n$ odd,

$$
=Q_{1}(\mu, \nu ; m, n)+Q_{2}(\mu, \nu ; m, n) \text {, }
$$$$
m \leqslant n, \quad m \text { even, } n \text { even, }
$$

$$
=Q_{2}(\mu, \nu ; m, n) \text {, }
$$$$
m>n, \quad m \text { even, } n \text { even, }
$$

$=0$, otherwise;

$Q_{1}(\mu, \nu ; m, n) \equiv \mu^{-(m+n+1) / 2} \nu^{(n-m) / 2} \frac{(-2)^{(m-n) / 2}(n !)^{1 / 2}}{[(n-m) / 2] !(m !)^{1 / 2}}$,

$$
\begin{aligned}
& Q_{2}(\mu, \nu ; m, n) \\
& \quad \equiv \mu^{-(m+n+1) / 2} \nu^{n / 2} \nu^{* m / 2}(-1)^{n} 2^{-(n+m) / 2} \frac{(n ! m !)^{1 / 2}}{(n / 2) !(m / 2) !} .
\end{aligned}
$$

Note that $m$ and $n$ must be both even or both odd in order that $\left\langle n \mid m_{g}\right\rangle$ be nonvanishing. Thus only an even number of photons would be counted for the state $\left|0_{g}\right\rangle=|0\rangle_{g}$. Other wave functions of $\left|m_{g}\right\rangle$ can be obtained accordingly. These wave functions provide the statistics of various measurements on the radiation states $|\beta\rangle_{g}$ and $\left|m_{g}\right\rangle$.

C. Quantum fluctuations and characteristic functions

From (3.1) and (3.2),

$$
a=\mu * b-\nu b^{\dagger},
$$

so that for the TCS $|\beta\rangle_{g}$,

$$
\begin{aligned}
& \langle a\rangle \equiv{ }_{g}\langle\beta|a| \beta\rangle_{g}=\mu^{*} \beta-\nu \beta^{*} \equiv \hat{\beta} \equiv \hat{\beta}_{1}+i \hat{\beta}_{2}, \\
& \left\langle a^{\dagger} a\right\rangle=|\hat{\beta}|^{2}+|\nu|^{2}, \\
& \left\langle\Delta a^{2}\right\rangle=-\mu^{*} \nu=\left\langle\Delta a^{\dagger 2}\right\rangle^{*}, \\
& \left\langle\Delta a_{1}^{2}\right\rangle=\frac{1}{4}|\mu-\nu|^{2}, \\
& \left\langle\Delta a_{2}^{2}\right\rangle=\frac{1}{4}|\mu+\nu|^{2}, \\
& \left\langle\Delta a_{1} \Delta a_{2}\right\rangle \equiv\left\langle\left(a_{1}-\hat{\beta}_{1}\right)\left(a_{2}-\hat{\beta}_{2}\right)\right\rangle=\frac{1}{4} i\left(\mu^{*} \nu-\nu^{*} \mu+1\right), \\
& \left\langle\Delta a_{2} \Delta a_{1}\right\rangle=\frac{1}{4} i\left(\mu^{*} \nu-\nu^{*} \mu-1\right)
\end{aligned}
$$

Equation (3.32) is, of course, consistent with the commutator

$$
\left[a_{1}, a_{2}\right]=\left[b_{1}, b_{2}\right]=\frac{1}{2} i \text {. }
$$

Compared to the case of a coherent state where

$$
\left\langle\alpha\left|(\Delta a)^{\dagger}(\Delta a)\right| \alpha\right\rangle=0,
$$

a TCS $|\beta\rangle_{g}$ has added quantum noise energy

$$
{ }_{g}\left\langle\beta\left|(\Delta a)^{\dagger}(\Delta a)\right| \beta\right\rangle_{g}=|\nu|^{2} \text {. }
$$

For self-adjoint $b_{1}, b_{2}$, and real $\beta_{1}, \beta_{2}$, let

$$
b \equiv b_{1}+i b_{2}, \quad \beta \equiv \beta_{1}+i \beta_{2} .
$$

The TCS $|\beta\rangle_{g}$ can be characterized as the states which minimize both $\left\langle\Delta b_{1}^{2}\right\rangle\left\langle\Delta b_{2}^{2}\right\rangle \geqslant \frac{1}{16}$ and $\left\langle\Delta b_{1}^{2}\right\rangle$ $+\left\langle\Delta b_{2}^{2}\right\rangle \geqslant \frac{1}{2}$. On the other hand, $|\beta\rangle_{g}$ minimizes the uncertainty product only when

$$
\mu=\delta \nu, \quad \text { for } \delta \text { real. }
$$

These minimum uncertainty states ${ }^{2}$ include the eigenstates of $a_{1}, a_{2}$ in the limit $\mu, \nu \rightarrow \infty$, such that (3.2) holds. Specifically, the eigenstates $\left|\alpha_{1}\right\rangle_{1}$ are obtained when $\delta \rightarrow 1$ and the eigenstates $\left|\alpha_{2}\right\rangle_{2}$ are obtained when $\delta--1$. As with the minimumuncertainty states, the mean-square quantum fluctuations in $a_{1}$ and $a_{2}$ can be exchanged in $|\beta\rangle_{g}$ from (3.31). On the other hand, they are fixed at $\frac{1}{4}$ for coherent states.

Thus for a mode of frequency $\omega$, a significant noise energy $\frac{1}{4} \hbar \omega$ is obtained if either of the quadrature components is measured in a coherent state, while $\left\langle\Delta a_{1}^{2}\right\rangle \ll \frac{1}{4}$ with corresponding large $\left\langle\Delta a_{2}^{2}\right\rangle$ can be obtained for measurement of $a_{1}$ in a TCS. 
A mathematical scaling of $a$ will, of course, lead to $\left\langle\Delta a_{1}^{2}\right\rangle<\frac{1}{4}$ even in an ordinary coherent state. For example, $\left\langle\Delta b_{1}^{2}\right\rangle<\frac{1}{4}$ is possible with $b$ given by (3.1). In actual applications such scaling cannot affect the system performance. In Sec. VII B we will show how the noise in a coherent signal can be reduced if $|\beta\rangle_{g}$ is available, which is impossible by scaling on $|\beta\rangle$. The fact is that $\left\langle\Delta a_{1}^{2}\right\rangle$ measures the absolute amount of noise in a quadrature component and $a$ is a more fundamental variable than $b$. See Secs. III E, IV A, and VII for further discussion and clarification.

From (3.20) one readily obtains

$$
\begin{aligned}
\left|\langle\alpha \mid \beta\rangle_{g}\right|^{2}=|\mu|^{-1} \exp [ & -\left(1-2 C_{1}\right)\left(\alpha_{1}-\hat{\beta}_{1}\right)^{2} \\
- & \left(1+2 C_{1}\right)\left(\alpha_{2}-\hat{\beta}_{2}\right)^{2} \\
+ & \left.4 C_{2}\left(\alpha_{1}-\hat{\beta}_{1}\right)\left(\alpha_{2}-\hat{\beta}_{2}\right)\right],
\end{aligned}
$$

where

$$
C \equiv-\nu / 2 \mu \equiv C_{i}+i C_{2}, \quad C_{1}, C_{2} \text { real, }
$$

and $\hat{\beta}$ is given by (3.28). From (3.2), $|C|^{2} \leqslant \frac{1}{4}$, so that

$$
\left|C_{1}\right| \leqslant \frac{1}{2}, \quad\left|C_{2}\right| \leqslant \frac{1}{2} .
$$

Thus (3.38) is indeed a Gaussian joint probability density function in $\left(\alpha_{1}, \alpha_{2}\right)$, with mean $\left(\hat{\beta}_{1}, \hat{\beta}_{2}\right)$ and variance

$$
\begin{aligned}
& \sigma_{1}^{2} \equiv E\left[\left(\alpha_{1}-\hat{\beta}_{1}\right)^{2}\right]=\frac{1}{2}\left(1+2 C_{1}\right) /\left(1-4|C|^{2}\right), \\
& \sigma_{2}^{2} \equiv E\left[\left(\alpha_{2}-\hat{\beta}_{2}\right)^{2}\right]=\frac{1}{2}\left(1-2 C_{1}\right) /\left(1-4|C|^{2}\right) .
\end{aligned}
$$

The notation $E[R]$ indicates the probabilistic average of a random variable $R$ with respect to its density function. ${ }^{31}$ The correlation coefficient is

$$
r \equiv E\left[\left(\alpha_{1}-\hat{\beta}_{1}\right)\left(\alpha_{2}-\hat{\beta}_{2}\right)\right] / \sigma_{1} \sigma_{2}=2 C_{2}\left(1-4 C_{1}\right)^{-1 / 2} .
$$

This density function is illustrated in Fig. 1.

Equation (3.38) is conveniently expressed in the matrix form

$$
\begin{aligned}
\rho_{g}^{(n)}\left(\alpha_{1}, \alpha_{2}\right) & \equiv\left|\langle\alpha \mid \beta\rangle_{g}\right|^{2} \\
& =|\mu|^{-1} \exp \left[-\frac{1}{2}\left(\underline{\alpha}_{\alpha}-\underline{\bar{\alpha}}_{\alpha}\right)^{T} \underline{\Lambda}^{-1}\left(\underline{\alpha}_{\alpha}-\underline{\bar{\alpha}}_{\alpha}\right)\right],
\end{aligned}
$$

where

$$
\begin{aligned}
& \underline{\alpha}_{q} \equiv\left(\begin{array}{c}
\alpha_{1} \\
\alpha_{2}
\end{array}\right), \quad \underline{\alpha}_{q}^{T} \equiv\left(\alpha_{1}, \alpha_{2}\right), \\
& \underline{\alpha}_{q} \equiv E\left[\underline{\alpha}_{q}\right]=\underline{\hat{\beta}}_{q}, \\
& \underline{\Lambda} \equiv \underline{\Lambda}_{s}+\frac{1}{4} \underline{I}, \quad \underline{I} \equiv\left(\begin{array}{cc}
1 & 0 \\
0 & 1
\end{array}\right),
\end{aligned}
$$

$$
\begin{aligned}
& \underline{\Lambda}_{s} \equiv\left(\begin{array}{cc}
\left\langle\Delta a_{1}^{2}\right\rangle & \frac{1}{2}\left\langle\Delta a_{1} \Delta a_{2}+\Delta a_{2} \Delta a_{1}\right\rangle \\
\frac{1}{2}\left\langle\Delta a_{1} \Delta a_{2}+\Delta a_{2} \Delta a_{1}\right\rangle & \left\langle\Delta a_{2}^{2}\right\rangle
\end{array}\right) \\
& \underline{\Lambda}^{-1}=2\left(\begin{array}{cc}
1-2 C_{1} & -2 C_{2} \\
-2 C_{2} & 1+2 C_{1}
\end{array}\right)
\end{aligned}
$$

In terms of the variables $\left(\alpha^{*}, \alpha\right)$, (3.38) becomes

$$
\rho_{g}^{(n)}\left(\alpha^{*}, \alpha\right)=|\mu|^{-1} \exp \left[-\frac{1}{2}\left(\underline{\alpha}_{a}-\underline{\alpha}_{a}\right)^{T} \underline{\Lambda}^{-1}\left(\underline{\alpha}_{a}-\underline{\bar{\alpha}}_{a}\right)\right]
$$

through the transformation

$$
\begin{aligned}
& \underline{\underline{\alpha}}_{a} \equiv\left(\begin{array}{c}
\alpha \\
\alpha^{*}
\end{array}\right)=\left(\begin{array}{cc}
1 & i \\
1 & -i
\end{array}\right)\left(\begin{array}{c}
\alpha_{1} \\
\alpha_{2}
\end{array}\right) \equiv \underline{\Omega} \underline{\alpha}_{q}, \\
& \underline{\Lambda}_{a} \equiv\left(\begin{array}{cc}
\left\langle\Delta a^{2}\right\rangle & \left\langle\Delta a \Delta a^{\dagger}\right\rangle \\
\left\langle\Delta a \Delta a^{\dagger}\right\rangle & \left\langle\Delta a^{\dagger}\right\rangle
\end{array}\right)=\underline{\Omega} \underline{\Omega^{T}},
\end{aligned}
$$

where $T$ denotes the transpose of a matrix.

The antinormally ordered characteristic function $\chi_{A}^{g}\left(\eta^{*}, \eta\right)$ for

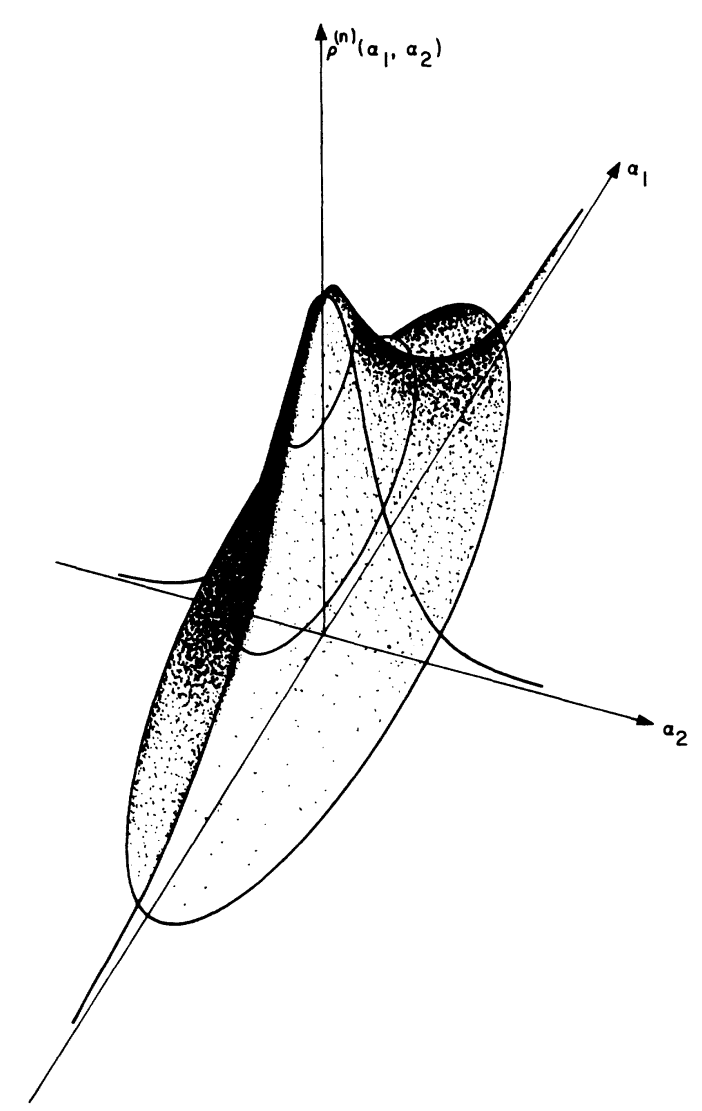

FIG. 1. Representation of a two-photon coherent state by $\rho^{(n)}\left(\alpha_{1}, \alpha_{2}\right)$ from (3.38), with $\sigma_{1}^{2}>\sigma_{2}^{2}$; a coherent state is obtained for $\sigma_{1}^{2}=\sigma_{2}^{2}$. 


$$
\rho_{g} \equiv|\beta\rangle_{g g}\langle\beta|
$$

is then

$$
\begin{aligned}
& \chi_{A}^{g}\left(\eta_{1}, \eta_{2}\right)=\exp \left(i \underline{\eta}_{q}^{T} \underline{\alpha}_{q}-\frac{1}{2} \underline{\eta}_{q}^{T} \underline{\Lambda} \underline{\eta}_{q}\right), \\
& \underline{\eta}_{q} \equiv\left(\begin{array}{c}
2 \eta_{2} \\
-2 \eta_{1}
\end{array}\right), \quad \eta \equiv \eta_{1}+i \eta_{2},
\end{aligned}
$$

and the Weyl characteristic function is, from (2.32),

$$
\chi_{W}^{g}\left(\eta_{1}, \eta_{2}\right)=\exp \left(i \underline{\eta}_{q}^{T} \underline{\bar{\alpha}}_{q}-\frac{1}{2} \underline{\eta}_{q}^{T} \underline{\Lambda}_{s} \underline{\eta}_{q}\right) .
$$

Since $\Lambda>0$ (positive definite), ${ }^{34}$ the Wigner distribution is also a Gaussian density function. The antinormally ordered characteristic function $\chi_{N}^{g}\left(\eta_{1}, \eta_{2}\right)$ is

$$
\chi_{N}^{g}\left(\eta_{1}, \eta_{2}\right)=\exp \left[i \underline{\eta}_{a}^{T} \underline{\bar{\alpha}}_{q}-\frac{1}{2} \underline{\eta}_{a}^{T}\left(\underline{\Lambda}-\frac{1}{4} \underline{I}\right) \underline{\eta}_{q}\right] .
$$

The antinormal-, symmetrized-, and normalorder moments of $\left(a^{\dagger}, a\right)$ can be calculated from (3.53), (3.55), and (3.56), respectively. Note that these $\chi_{N}, \chi_{W}, \chi_{A}$, and $W, \rho^{(n)}$ for TCS's are all Gaussian. The above matrix forms are convenient for generalization to the multimode situations.

The minimum noise $\left\langle\Delta b_{1}^{2}\right\rangle$ obtainable in a TCS for a self-adjoint $b_{1}$,

$$
b_{1} \equiv \gamma_{1} a_{1}+\gamma_{2} a_{2}, \quad \gamma_{1}^{2}+\gamma_{2}^{2}=1,
$$

is the minimum eigenvalue of $\Lambda_{s}$, with the minimizing value of $\left(\gamma_{1}, \gamma_{2}\right)$ given by the corresponding eigenvector..$^{35}$ The result can be expressed in the following form: Let

$$
\gamma_{1}+i \gamma_{2} \equiv e^{-i \phi}
$$

for a real $\phi$ and let

$$
a^{\prime} \equiv a e^{i \Phi} \text {. }
$$

Then $a_{1}^{\prime}$ minimizes $\left\langle\Delta b_{1}^{2}\right\rangle$ when

$$
\phi=\tan ^{-1}\left[i\left(\mu^{*} \nu-\nu^{*} \mu\right) /\left(2|\mu||\nu|+\mu^{*} \nu+\nu^{*} \mu\right)\right]
$$

and the minimum fluctuation is

$$
\left\langle\Delta a_{1}^{\prime 2}\right\rangle=\frac{1}{4}(|\mu|-|\nu|)^{2} .
$$

The conjugate variable $a_{2}^{\prime}$ is the one which maximizes $\left\langle\Delta b_{2}^{2}\right\rangle$, with

$$
\left\langle\Delta a_{2}^{\prime 2}\right\rangle=\frac{1}{4}(|\mu|+|\nu|)^{2} .
$$

From (3.61), the TCS $|\beta\rangle_{g}$ can be seen to minimize the uncertainty product

$$
\left\langle\Delta a_{1}^{\prime 2}\right\rangle\left\langle\Delta a_{2}^{\prime 2}\right\rangle \geqslant \frac{1}{16},
$$

for $a^{\prime}$ defined by (3.59) and (3.60). Thus $|\beta\rangle_{g}$ are the minimum-uncertainty-product states for $a^{\prime}$ and can therefore be understood as generalized or rotated minimum-uncertainty states. This fact is important in some applications as indicated in Sec. VII B, because the original $\left\langle\Delta a_{1}^{2}\right\rangle$ and $\left\langle\Delta a_{2}^{2}\right\rangle$ can be both larger than $\frac{1}{4}$ from (3.31) unless $\mu / \nu$ is real.

D. Diagonal operator representation for $|\beta\rangle_{g}\langle\beta|$

Let $\rho_{+}$represent a density operator which can be written as a random superposition of coherent states, i.e.,

$$
o_{+}=\int P_{+}\left(\alpha^{*}, \alpha\right)|\alpha\rangle\langle\alpha| d^{2} \alpha,
$$

for a positive true probability density function $P_{+}\left(\alpha^{*}, \alpha\right)$. It follows that

$$
\operatorname{tr} \rho_{+}\left(\Delta a_{1}^{2}\right)=\frac{1}{4}+\int P_{+}\left(\alpha_{1}, \alpha_{2}\right)\left(\alpha_{1}-\bar{\alpha}_{1}\right)^{2} d \alpha_{1} d \alpha_{2} \geqslant \frac{1}{4},
$$

for

$$
\bar{\alpha}_{1}=\int P_{+}\left(\alpha_{1}, \alpha_{2}\right) \alpha_{1} d \alpha_{1} d \alpha_{2},
$$

and similarly,

$$
\operatorname{tr} \rho_{+}\left(\Delta a_{2}^{2}\right) \geqslant \frac{1}{4} \text {. }
$$

Equation (3.64) states the obvious physical fact that the introduction of classical randomness merely increases the noise in $a_{1}$ and $a_{2}$.

A TCS with (3.31) therefore cannot be a mixture of coherent states. In fact, the density operator $\rho_{g}$ cannot be written in the diagonal form (2.17) with $P\left(\alpha^{*}, \alpha\right)$ belonging to the class of tempered distribution. This can be seen from the normally ordered characteristic function $\chi_{N}^{g}\left(\eta_{1}, \eta_{2}\right)$. For a TCS, $\underline{\Lambda}-\frac{1}{4} I$ cannot be positive definite. Thus $\chi_{N}^{g}$ does not possess a Fourier transform. If we insist on having a diagonal $P$ representation for $\boldsymbol{\rho}_{g}$, we will need the Fourier transform of $e^{\gamma x^{2}}$ for a real variable $x$ and $\gamma>0$. It does not appear to be useful to talk about the Fourier transform of such a quantity, which certainly does not belong to the class of tempered distribution. Further discussion of this divergence is given in Ref. 27.

One may still consider the diagonal $P$ representation of $\rho_{g}$ as belonging to the class of distribution $Z_{2},{ }^{24}$ or alternatively as the limit of a sequence of well-behaved density functions. ${ }^{23}$ Since no useful method of calculation has resulted from such viewpoints and since $\rho^{(n)}\left(\alpha^{*}, \alpha\right)$ is sufficient for our purpose, we will say that the $P$ representation of $\rho_{g}$ does not exist and we will not employ it in the following.

Note, however, that a generalized $P_{g}$ representation of $\rho_{g}$ does exist and can be useful. Thus

$$
\rho=\int P_{g}\left(\beta^{*}, \beta\right)|\beta\rangle_{g}\langle\beta| d^{2} \beta / \pi
$$

may be used to represent $|\bar{\beta}\rangle_{g}$, with 


$$
P_{g}\left(\beta^{*}, \beta\right)=\delta^{2}(\beta-\bar{\beta}) .
$$

Equation (3.66) also describes random mixtures of $|\beta\rangle_{g}$ in the same way as (3.63). In particular, it remains useful with the introduction of an additive noise. However, it is inconvenient if $(\mu, \nu)$ are time varying. While it can be used in moment calculations by expressing $M\left(a^{\dagger}, a\right)$ in normalorder form $M^{(n)}\left(b^{\dagger}, b\right)$ in terms of the variables $\left(b^{\dagger}, b\right)$, it is no longer particularly suited to be calculations of $\left(a^{\dagger}, a\right)$ normal-order moments encountered in the description of most experimental results. Therefore (3.65) will also not be employed in this paper.

\section{E. Coherent states versus two-photon coherent states}

As mentioned in Sec. I, two-photon coherent states were encountered in many different studies, but they received little attention in quantum optics. Obviously this is due largely to the fact that these states are not generated from available optical sources, but other factors may have also contributed.

It is well known that the mathematical form of a minimum-uncertainty state at a frequency $\omega_{1}$ is identical to that of a coherent state at a different frequency $\omega_{2}$. Therefore one may tend to view minimum-uncertainty states as just coherent states of a different frequency, particularly since the frequency is often normalized away. This viewpoint is erroneous, because in the state space $\mathfrak{F C}\left(\omega_{1}\right) \otimes \mathfrak{F C}\left(\omega_{2}\right)$ a minimum-uncertainty state at $\omega_{1}$ is given by $|\beta\rangle_{g} \otimes|0\rangle$, while a coherent state at $\omega_{2}$ is $|0\rangle \otimes|\beta\rangle$. Physically, no photocounts would be registered for a coherent state at $\omega_{2}$ in a counting experiment employing detectors responding only to fields at $\omega_{1}$. In this connection one may also note that for a fixed mode the frequency is also fixed by the dispersion relation. Changing the frequency of a fixed mode by dielectric modulation would not yield a minimum-uncertainty state at frequency $\omega_{1}$ from a coherent state at frequency $\omega_{1}$. A coherent state at a different frequency $\omega_{2}$ would be obtained instead.

Alternatively, one may regard TCS's and ordinary coherent states as (mathematically) equivalent, since they are related through a unitary transformation or some kind of scaling. The question of scaling was already dealt with in Sec. III C concerning the noise in quadrature components. It is true that two-photon coherent states are coherent states in a broad sense: they are the coherent states with respect to $b(\nu \neq 0)$ instead of $a$. However, this abstract unitary equivalence means little in describing actual experimental results. Thus $\left\langle n_{g} \mid \alpha\right\rangle_{g}=\langle n \mid \alpha\rangle$ but $\langle n \mid \alpha\rangle_{g}$ has little resemblance to $\langle n \mid \alpha\rangle$. In general, for an arbitrary uni- tary transformation $U$ we can define a set of states |\rangle

$$
|\rangle=U|\alpha\rangle \text {. }
$$

The functions $\langle x \mid\rangle$ cannot be obtained by simple scaling on $\langle x \mid \alpha\rangle$ and has to be computed anew via $\langle x|U| \alpha\rangle$. This paper develops such specific results for the $U_{L}$ of (3.5).

With respect to $a$, whose zero eigenstate is the radiation ground state occurring in nature, the properties of $|\alpha\rangle_{g}$ are quite different from $|\alpha\rangle$ in many respects, as presented in this section. Since the term coherent state is usually understood in the original sense (2.1), a new terminology is required for finer distinction between coherent states in the broad sense. The term two-photon coherent states ${ }^{36}$ is adopted because of the close connection between these states and quadratic Hamiltonians or two-photon lasing processes, discussed below.

\section{QUADRATIC HAMILTONIAN AND STRUCTURE OF $U_{L}$}

From (3.11) and (3.20) we can see that $U_{L}^{(n)}\left(\alpha^{*}, \alpha\right)$ is an exponentiation of a quadratic form in $\left(\alpha^{*}, \alpha\right)$. Such a $U_{L}^{(n)}\left(\alpha^{*}, \alpha\right)$ can be written as $\left\langle\alpha\left|e^{i M}\right| \alpha\right\rangle$ for a self-adjoint operator $M$ quadratic in $\left(a^{\dagger}, a\right)$, from a theorem of McCoy. ${ }^{37}$ Therefore $U_{L}$ must be the unitary operator generated by a quadratic Hamiltonian $H_{Q}$. The mathematical solution $U_{Q}\left(t, t_{0}\right)$ of the Schrödinger equation

$$
i \hbar \frac{\partial U_{Q}}{\partial t}=H_{Q} U_{Q}, \quad U_{Q}\left(t_{0}, t_{0}\right)=1,
$$

is investigated in some detail in this section, which gives all of the $U_{L}$.

\section{A. Positive definite quadratic Hamiltonian}

Consider the general quadratic Hamiltonian

$$
H_{Q}=\hbar\left(f_{1} a^{\dagger} a+f_{2}^{*} a^{2}+f_{2} a^{\dagger 2}+f_{3}^{*} a+f_{3} a^{\dagger}\right),
$$

where the $c$ numbers $f_{i}$ may be time dependent. From Hermiticity $f_{1}$ has to be real. For a mode of frequency $\omega$, we have $f_{1}=\omega$, and $\hbar f_{1} a^{\dagger} a$ represents the free radiation energy of that mode. The $f_{2}$ and $f_{3}$ terms then represent interaction energies. In particular, the $f_{2}$ terms describe a two-photon mechanism, whereas the $f_{3}$ terms describe the usual one-photon or linear driving mechanism.

If a physical system with Hamiltonian given by (4.2) is stable, $H_{Q}$ should not have a spectrum ranging through the entire continuum from $-\infty$ to $\infty$ in order for a ground state to be defined, i.e., the spectrum of $H_{Q}$ should be lower bounded. By a shift of scale one may require that the Hamiltonian be positive definite. ${ }^{38}$ If this condition is not satisfied, (4.2) cannot be properly used to de- 
scribe a physical system of interest in our present context. It has to be extended to incluaie a more complete description of other relevant jactors of importance.

Under the condition

$$
f_{1}>2\left|f_{2}\right|
$$

the Hamiltonian (4.2) can be changed, similar to the Bogoliubov transformation, ${ }^{39}$ to the following form:

$$
\begin{aligned}
& H_{Q}=f_{0} b^{\dagger} b+f_{0}\left(\left|\mu f_{3}^{*}-\nu f_{3}\right|^{2}+|\nu|^{2}\right), \\
& \boldsymbol{b} \equiv \mu a+\nu a^{\dagger}+\mu f_{3}^{*}-\nu f_{3}, \\
& \mu \equiv\left[2 / f_{0}\left(f_{1}-f_{0}\right)\right]^{1 / 2} f^{*} e^{i \phi}, \\
& \nu \equiv\left[\left(f_{1}-f_{0}\right) /\left.f_{0}\right|^{1 / 2} e^{i \phi},\right. \\
& f_{0} \equiv\left(f_{1}^{2}-4\left|f_{2}\right|^{2}\right)^{1 / 2},
\end{aligned}
$$

where $\phi$ is an arbitrary phase. The canonical transformation (4.5) is an inhomogeneous linear one, with (4.6) obeying (3.2). The Hamiltonian (4.4) is manifestly positive definite, and (4.3) is indeed the necessary and sufficient condition for a classical $H_{Q}$ of the form (4.2) to be positive definite.

Similar to the situation of an interacting Bose fluid, $b^{\dagger}$ of (4.5) can be interpreted as the quasiboson creation operator with a quasiparticle spec$\operatorname{trum} f_{0}$. The quasiparticle ground state is the $\left|0_{g}\right\rangle$ of (3.7), whereas $\left|m_{g}\right\rangle$ are the excited states. It seems highly unlikely, however, that the radiation states $\left|m_{g}\right\rangle$ may be generated optically.

The following observation should be made: A linear canonical transformation from $a$ to $b$ can always produce $f_{2}$ terms in $b$ from a free Hamiltonian $h f_{1} a^{\dagger} a$. In fact, it is easily checked that an $f_{2}$ term results if and only if the $\nu$ term mixing $b^{\dagger}$ is present in the transformation. The transformed Hamiltonian contains a constant term $|\nu|^{2}$, so that the eigenstates of $b$ have this added excitation energy compared to the true ground state $|0\rangle$. Such a Hamiltonian is clearly not physically equivalent to (4.2). In this sense the operator $a$ is more fundamental than $b$ and further justifies the need for viewing $|\alpha\rangle_{g}$ as a two-photon coherent state with respect to $a$.

Our following results on $U_{Q}\left(t, t_{0}\right)$ are actually valid independent of the condition (4.3). Nevertheless, one should note the significance of the positive definiteness of (4.2).

$$
\text { B. Explicit solution for } U_{Q}\left(t, t_{0}\right)
$$

The solution $U_{Q}\left(t, t_{0}\right)$ can be obtained by normalordering techniques, ${ }^{3}$ among many possible methods. With (4.2), the solution $U_{Q}^{(n)}\left(\alpha^{*}, \alpha ; t, t_{0}\right)$ of (2.16) is of the form

$$
\begin{aligned}
& U_{Q}^{(n)}\left(\alpha^{*}, \alpha ; t, t_{0}\right)=\exp [ A(t)+B(t) \alpha^{2}+C(t) \alpha^{* 2} \\
&\left.+D(t)|\alpha|^{2}+E(t) \alpha+F(t) \alpha^{*}\right],
\end{aligned}
$$

where

$$
\begin{aligned}
& \frac{d A}{d t}=-2\left(2 f_{2}^{*} C+f_{2}^{*} F^{2}+f_{3}^{*} F\right), \\
& \frac{d B}{d t}=-i f_{2}^{*}(D+1)^{2}, \\
& \frac{d C}{d t}=-2\left(4 f_{2}^{*} C^{2}+2 f_{1} C+f_{2}\right), \\
& \frac{d D}{d t}=-i\left(4 f_{2}^{*} C+f_{1}\right)(D+1), \\
& \frac{d E}{d t}=-i\left(2 f_{2}^{*} F+f_{3}^{*}\right)(D+1), \\
& \frac{d F}{d t}=-i\left[\left(4 f_{2}^{*} C+f_{1}\right) F+2 f_{3}^{*} C+f_{3}\right],
\end{aligned}
$$

with the initial condition

$$
A\left(t_{0}\right)=B\left(t_{0}\right)=C\left(t_{0}\right)=D\left(t_{0}\right)=E\left(t_{0}\right)=F\left(t_{0}\right)=0 .
$$

From (4.8) and (2.14) we get

$$
\begin{aligned}
\left\langle\alpha\left|U_{Q}\left(t, t_{0}\right)\right| \beta\right\rangle=\exp & \left\{-\frac{1}{2}|\alpha|^{2}-\frac{1}{2}|\beta|^{2}+A(t)+B(t) \beta^{2}\right. \\
& +C(t) \alpha^{* 2}+[D(t)+1] \alpha^{*} \beta \\
& \left.+E(t) \beta+F(t) \alpha^{*}\right\} .
\end{aligned}
$$

Equation (4.11) can be written in the form of $\langle\alpha \mid \beta+\zeta(t) ; \mu(t) \nu(t)\rangle$, apart from a phase factor. Using (3.20), we find by comparison that

$$
\begin{aligned}
& U_{Q}\left(t, t_{0}\right)|\beta\rangle=|\beta+\zeta(t) ; \mu(t), \nu(t)\rangle e^{i \phi(t)}, \\
& \phi(t) \equiv \Theta(t)+\frac{1}{2} i\left[\beta^{*} \zeta(t)-\beta \zeta^{*}(t)\right],
\end{aligned}
$$

for a real-valued $(\beta, \zeta)$ independent function $\Theta(t)$ and

$$
\begin{aligned}
A(t)= & -\frac{1}{2} \ln \mu(t)+\nu^{*}(t) \zeta^{2}(t) / 2 \mu(t) \\
& \left.-\frac{1}{2}|\zeta(t)|^{2}+\imath \theta t\right), \\
B(t)= & \nu^{*}(t) / 2 \mu(t), \\
C(t)= & -\nu(t) / 2 \mu(t), \quad D(t)=1 / \mu(t)-1, \\
E(t)= & \nu^{*}(t) \mu(t) / \zeta(t)-\zeta^{*}(t), \quad F(t)=\zeta(t) / \mu(t) .
\end{aligned}
$$

If (4.14) is substituted into (4.9), the equations of motion for $\mu, \nu, \zeta$, and $\Theta$ are obtained,

$$
\begin{aligned}
& \frac{d \mu}{d t}=i f_{1} \mu-2 i f_{2}^{*} \nu, \frac{d \nu}{d t}=-i f_{1} \nu+2 i f_{2} \mu, \\
& \frac{d \zeta}{d t}=i\left(f_{3}^{*} \nu-f_{3} \mu\right),
\end{aligned}
$$




$$
\frac{d \Theta}{d t}=\frac{1}{2} f_{1}+\frac{i}{2}\left[\zeta\left(\frac{d \zeta^{*}}{d t}\right)-\zeta^{*}\left(\frac{d \zeta}{d t}\right)\right]
$$

Equations (4.15)-(4.17) are equivalent to (4.9). The initial condition (4.10) becomes

$$
\begin{aligned}
& \quad \mu(0)=1, \quad \nu(0)=0, \\
& \zeta(0)=0, \\
& \text { while } \\
& \qquad(0)=0
\end{aligned}
$$

is obtained from (4.12). The general solution (4.8) or (4.12) can be given in quadrature once the solution for (4.15) is given in quadrature, since the other equations can then be immediately integrated. Defining

$$
\begin{aligned}
& \mathcal{F}\left(t, t_{0}\right) \equiv \int_{t_{0}}^{t} f_{1}(\tau) d \tau, \\
& f \equiv 2 i f_{2} e^{2 i \mathcal{F}\left(t, t_{0}\right)},
\end{aligned}
$$

the solution of the first-order coupled differential equation (4.15) under (4.18) is equivalent to the solution of the following equation for a new variable $\mathfrak{e}$ :

$$
\begin{aligned}
& \frac{d \mathfrak{C}}{d t}=f^{*} \mathfrak{C}^{2}-f, \mathfrak{e}(0)=0, \\
& \mathbb{C} \equiv 2 \mathbb{C} e^{2 i \mathcal{F}\left(t, t_{0}\right)} .
\end{aligned}
$$

Once $C$ is obtained through (4.23) and (4.24), all of the other variables $A, B, D, E$, and $F$ can be given in quadratures from (4.19). But Eq. (4.23), a case of the well-studied Riccati equation, does not admit a solution in quadrature in general, although many methods and results on its solution are available.$^{40,41}$

The existence and uniqueness of a solution to (4.23) imply that the solution of (4.9) and (4.10) also exists and is unique. Thus (4.8) is indeed the solution we seek. With $U^{(n)}\left(\alpha^{*}, \alpha\right)$ given by $(4.8)$, the explicit operator expression of $U_{Q}\left(t, t_{0}\right)$ is

$$
U\left(t, t_{0}\right)=e^{A(t)} e^{C(t) a^{\dagger 2}+F(t) a^{\dagger}} e^{\ln [D(t)+1] a^{\dagger} a} e^{B(t) a^{2}+E(t) a} .
$$

Equation (4.25) can also be written as

$$
\exp \left(\gamma_{1} a^{\dagger 2}+\gamma_{2} a^{\dagger} a+\gamma_{3} a^{2}+\gamma_{4} a^{\dagger}+\gamma_{5} a\right)
$$

for a set of $c$ numbers $\gamma_{i} \cdot{ }^{37}$

\section{Examples}

Equation (4.23) can be integrated when $f / f^{*}$ is independent of $t$, i.e., when

$$
f_{2}(t)=r(t) \exp \left[i \phi_{2}-2 i \mathcal{F}\left(t, t_{0}\right)\right],
$$

$\phi_{2}$ independent of $t$, for a real-valued function $r(t)$ which can take on both positive and negative values. Under (4.26), one finds readily from (4.23), (4.24), $(4.9 \mathrm{~d}),(4.14 \mathrm{c})$, and $(4.18)$ that

$$
\begin{aligned}
& \mu(t)=e^{i \Im\left(t, t_{0}\right)} \cosh [2 \mathcal{R}(t)], \\
& \nu(t)=i e^{i \Phi_{2}-i \mathcal{F}_{\left(t, t_{0}\right)} \sinh [2 \mathcal{R}(t)],} \\
& \mathcal{R}(t) \equiv \int_{t_{0}}^{t} r(\tau) d \tau, \\
& \zeta(t)=i \int_{t_{0}}^{t}\left(f_{3}^{*} \nu-f_{3} \mu\right) d t .
\end{aligned}
$$

Equation (4.26) is especially interesting because of the resonant factor $e^{-2 i \omega\left(t-t_{0}\right)}$ obtained when $f_{1}$ $=\omega$. This factor is automatically present in our later physical interpretation of (4.2). The noise behavior in this case is of great interest. Thus when (with also $f_{1}=\omega, t_{0}=0$ )

$$
\phi_{2}=\frac{1}{2} \pi \text {, }
$$

$r=$ const independent of $t$, we have from $(3.61)$ and $(4.27)$

$$
\left\langle\Delta a_{1}^{\prime 2}(t)\right\rangle=\frac{1}{4} e^{-4 r t}, \quad\left\langle\Delta a_{2}^{\prime 2}(t)\right\rangle=\frac{1}{4} e^{4 r t},
$$

for $a^{\prime}=a e^{i \omega t}$. In this case the noise in one quadrature component, apart from the optical oscillation, decreases exponentially while the conjugate component has exponentially increasing noise. The quadrature components $a_{1}$ and $a_{2}$ are completely symmetrical here. We can choose the sign of $r$, or equivalently the phase of $r$, to cause either one of them to have decreasing noise. For $\phi_{2}=0, r=$ const, we again have (4.31) with $a^{\prime}$ $=a e^{i(\omega t-\pi / 4)}$. The nature of phase control in $a^{\prime}$ from the phase of $f_{2}$ should be clear in this example.

Equation (4.15) can be integrated for a constant $f_{2}$ independent of time, with

$$
\begin{aligned}
& \mu(t)=\cos (\lambda t)+(i \omega / \lambda) \sin (\lambda t), \\
& \nu(t)=i\left(2 f_{2} / \lambda\right) \sin (\lambda t),
\end{aligned}
$$

for $\lambda \equiv\left(\omega^{2}-4\left|f_{2}\right|^{2}\right)^{1 / 2} \geq 0$. The quantum noise is purely oscillatory in this situation, without attenuation in time.

Equation (4.15) or (4.23) may be integrated in many other situations. A way of generating such example is discussed below.

$$
\text { D. Properties of } U_{Q}\left(t, t_{0}\right)
$$

A number of general features exhibited by $U_{Q}\left(t, t_{0}\right)$ are now observed. From (4.15) we have

$$
\mu^{*} \frac{d \mu}{d t}=\nu \frac{d \nu^{*}}{d t}+i f_{1}
$$

which is a complex equation. The real part of this equation is implied by $(3.2)$, but the imaginary part 
is an additional constraint on the pair $\mu(t), \nu(t)$ obtainable from an $H_{Q}$. Thus not every pair of functions $\mu(t), \nu(t)$ obeying (3.2) can occur in a $U_{Q}\left(t, t_{0}\right)$. Only those obeying (4.33) are permitted in the form (4.12). However, this does not impose any constraint on the mean-square fluctuation behavior obtainable in (3.61), since only $|\mu|,|\nu|$ are involved here. Moreover, (4.27) shows that any particular pair of values $(\mu, \nu)$ obeying (3.2) can be obtained at a specific $t$ with a proper choice of $f_{2}$, even under (4.3) with $f_{1}$ fixed.

Any desirable pair of functions $\mu(t), \nu(t)$ obeying (4.33) and (3.2) can be generated by an $f_{2}$ through (4.15). This corresponds to the solution of the Riccati equation (4.23) for various $f$. For example, we can assume $\left(f_{1}=\omega, t_{0}=0\right)$

$$
\mu(t)=e^{i \Phi(t)} \cosh (\gamma t), \quad \nu(t)=e^{i \Phi(t)} \sinh (\gamma t),
$$

and determine the phase $\phi(t)$ so that (4.33) is satisfied. Equation (4.34) has the interesting characteristic that

$$
\left\langle\Delta a_{1}^{2}(t)\right\rangle=\frac{1}{4} e^{-2 \gamma t}, \quad\left\langle\Delta a_{2}^{2}(t)\right\rangle=\frac{1}{4} e^{2 \gamma t},
$$

without any shift of phase to $a^{\prime}$. Substitution of (4.34) into (4.33) shows that $\phi(t)$ obeys the differential equation

$$
\frac{d \phi}{d t}=\frac{1}{\cosh ^{2}(\gamma t)+\sinh ^{2}(\gamma t)},
$$

so that

$$
\phi(t)=(\omega / \gamma) \tan ^{-1}[\tanh (\gamma t)] .
$$

The corresponding $f_{2}(t)$ is given by

$$
f_{2}=\frac{1}{2} \tanh (2 \gamma t)-i \gamma / 2 \omega \text {. }
$$

Any arbitrary state $\left|\psi_{0}\right\rangle$ will evolve into

$$
\left|\psi_{t}\right\rangle=U_{Q}\left(t, t_{0}\right)\left|\psi_{0}\right\rangle=\int U_{Q}\left(t, t_{0}\right)|\beta\rangle\left\langle\beta \mid \psi_{0}\right\rangle d^{2} \beta / \pi
$$

under $U_{Q}$. If $\left|\psi_{0}\right\rangle=\left|\beta_{0} ; \mu_{0}, \nu_{0}\right\rangle,(4.38)$ can be readily determined from (4.12),

$$
\begin{aligned}
& U_{Q}\left(t, t_{0}\right)\left|\beta_{0} ; \mu_{0}, \nu_{0}\right\rangle \\
& =\left|\beta_{0}+\zeta\left(t, t_{0}\right) ; \mu\left(t, t_{0}\right), \nu\left(t, t_{0}\right)\right\rangle e^{i \Phi\left(t, t_{0}\right)}, \\
& \phi\left(t, t_{0}\right) \equiv \Theta(t)+\frac{1}{2} i\left[\beta^{*} \zeta\left(t, t_{0}\right)-\beta \zeta^{*}\left(t, t_{0}\right)\right], \\
& \mu\left(t, t_{0}\right)=\mu_{0} \mu(t)+\nu_{0} \nu^{*}(t), \\
& \nu\left(t, t_{0}\right)=\mu_{0} \nu(t)+\nu_{0} \mu^{*}(t), \\
& \delta\left(t, t_{0}\right)=\mu_{0} \zeta(t)+\nu_{0} \zeta^{*}(t),
\end{aligned}
$$

and $\mu, \nu, \zeta$, and $\Theta$ in $(4.40)-(4.42)$ are the solutions to $(4.15)-(4.20)$.
Therefore, under an arbitrary $U_{Q}\left(t, t_{0}\right)$ a TCS will remain a TCS for all time, but with $\beta, \mu$, and $\nu$ time varying. By solving $\mu(t), \nu(t)$, and $\zeta(t)$ in terms of $\mu_{0}$ and $\nu_{0}$ and $\mu\left(t, t_{0}\right), \nu\left(t, t_{0}\right)$, and $\delta\left(t, t_{0}\right)$, (4.39)-(4.42) and (4.27) show explicitly that any $|\beta ; \mu, \nu\rangle$ can be obtained from any given $\left|\beta_{0} ; \mu_{0}, \nu_{0}\right\rangle$ by a $U_{Q}\left(t, t_{0}\right)$.

It also follows from (4.41) that if $f_{2}$ is turned off at time $t$, then

$$
\mu(t)=\mu\left(t_{f}\right) e^{i \mathfrak{F}\left(t, t_{f}\right)}, \quad \nu(t)=\nu\left(t_{f}\right) e^{i \Im\left(t, t_{f}\right)} .
$$

Thus apart from the optical oscillation the values $\mu$ and $\nu$ are invariant in the absence of two-photon interaction in $H_{Q}$. Furthermore, such invariance is obtained when and only when

$$
f_{2}=0 \text {, }
$$

i.e., $\nu(t)=0$. In general, $\mu(t)$ and $\nu(t)$ are determined only by $f_{2}(t)$ independently of $f_{3}(t)$, as seen from (4.15). This is in accordance with the fact that the new quantum noise behavior is entirely a consequence of $f_{2} \neq 0$. Such correlation between $f_{2}$ and $\nu$ is also observed in Sec. IV A.

On the other hand, the added value $\zeta(t)$ depends on both $f_{2}$ and $f_{3}$ but is always zero for $f_{3}=0$,

$$
U_{Q}\left(t, t_{0}\right)|\beta\rangle=|\beta\rangle_{g} \text {, when } f_{3}=0 \text {. }
$$

Therefore all of the $U_{L}$ 's are generated by $H_{Q}$ with $f_{3}=0$. The addition of $f_{3}$ merely introduces a constant shift to $b$, as in (4.5). The states (4.39) are eigenstates of

$$
\boldsymbol{b}(t) \equiv \mu(t) a\left(t_{0}\right)+\nu(t) a^{\dagger}\left(t_{0}\right)
$$

where $a\left(t_{0}\right)$ is the Schrödinger-picture fixed photon annihilation operator. We have

$$
\boldsymbol{b}(t)=U_{Q}\left(t, t_{0}\right) a\left(t_{0}\right) U_{Q}^{\dagger}\left(t, t_{0}\right)+\zeta(t)=U_{L} a U_{L}^{\dagger} .
$$

Equation (4.47) also shows

$$
\mu(t) a_{H}(t)+\nu(t) a_{H}^{\dagger}(t)=a\left(t_{0}\right)+\zeta(t)
$$

for the Heisenberg operator

$$
a_{H}(t) \equiv U_{Q}^{\dagger}\left(t, t_{0}\right) a\left(t_{0}\right) U_{Q}\left(t, t_{0}\right) .
$$

Equation (4.48) implies, by solving for $a_{H}(t)$

$$
\begin{aligned}
& a_{H}(t)=\mu^{*}(t) a\left(t_{0}\right)-\nu(t) a^{\dagger}\left(t_{0}\right)-\zeta^{\prime}(t), \\
& \zeta^{\prime}(t) \equiv \nu(t) \zeta^{*}(t)-\mu^{*}(t) \zeta(t) .
\end{aligned}
$$

Equations (4.49) and (4.39) give

$$
U_{Q}^{\dagger}\left(t, t_{0}\right)\left|\beta_{0} ; \mu_{0}, \nu_{0}\right\rangle=\left|\beta_{0}+\zeta_{r}(t) ; \mu_{r}(t), \nu_{r}(t)\right\rangle e^{i \Phi_{r}(t)} \text {, }
$$

$\phi_{r}(t) \equiv-\Theta(t)+\frac{1}{2} i\left[\beta^{*} \zeta_{r}(t)-\beta \zeta_{r}^{*}(t)\right]$,

$\mu_{r}(t)=\mu_{0} \mu^{*}(t)-\nu_{0} \nu^{*}(t), \quad \nu_{r}(t)=\nu_{0} \mu(t)-\mu_{0} \nu(t)$,

$\zeta_{r}(t)=\mu_{0} \zeta^{\prime}(t)+\nu_{0} \zeta^{\prime *}(t)$ 
where $\mu, \nu, \zeta$, and $\theta$ are still the solution to (4.15)-(4.20). The state $U_{Q}^{\dagger}\left(t, t_{0}\right)|\beta\rangle_{g}$ is obtained from evolution of the system backwards in time under the same $f_{i}(t)$ in $H_{Q}$, and the operator $b(t)$ is similarly the $a_{H}(t)$ moving backwards, apart from a constant.

Even though no solution of $U_{Q}\left(t, t_{0}\right)$ in quadrature can be obtained in general, its structure and behavior have already been fully determined. These behaviors are important in the applications of TCS's.

\section{STATE OF TWO-PHOTON LASING RADIATION}

We suggest that the output radiation of an ideal monochromatic two-photon laser is in a TCS. In contrast, the output of an ideal one-photon laser is in an ordinary coherent state. Thus TCS's are the characteristic states of two-photon lasing processes (two photons from the same mode).

\section{A. Two-photon laser}

Two photons of the same frequency $\omega$ from the same radiation mode can be absorbed in a single atomic transition between two levels 2 and 1 via an intermediate state, i.e., a transition second or$\operatorname{der}$ in $\overrightarrow{\mathrm{p}} \cdot \overrightarrow{\mathrm{A}}$. Under the usual dipole approximation the parities of levels 2 and 1 have to be identical. First-order dipole transitions between the two levels are forbidden in this circumstance. On the other hand, higher-order multipole contributions to second-order $\overrightarrow{\mathrm{p}} \cdot \overrightarrow{\mathrm{A}}$ transitions, which may connect states of different parity, can be significant. ${ }^{42}$ First-order transitions between states of opposite parity are also possible via the $A^{2}$ term in the radiation interaction Hamiltonian. ${ }^{42-44}$ This is possible because in the multipole expansion of the vector potential $A(\overrightarrow{\mathbf{r}})$, higher-order multipole terms introduce atomic coordinates into the $A^{2}$ Hamiltonian. Two-photon absorption spectroscopy is an important experimental technique by now, ${ }^{45}$ and many discussions of two-photon absorption processes can be found in the literature.

The radiation-matter (to be referred to as atoms) interaction responsible for the two-photon transition can be expressed in the form

$$
H_{I}=\hbar\left(p M a^{\dagger 2}+p^{*} M^{\dagger} a^{2}\right),
$$

where $p$ is a coupling coefficient and $M$ is the atomic polarization operator which flips the state of the atom If the $A^{2}$ mechanism is under consideration, $H_{I}$ is a true Hamiltonian..$^{42-44}$ If virtual transition through an intermediate state has been suppressed through a nonlinear susceptibility, or equivalently through the coupling coefficient $p$, (5.1) is only an effective Hamiltonian. In this case it can be derived by following a procedure similar to the standard quantum theory of parametric interaction. ${ }^{4,47}$ By methods widely used in nonlinear optics, ${ }^{7}$ classical equations of motion for the field and the active atomic variables can be obtained in which the virtual intermediate states are accounted for through $p$. Examples of such treatment for twophoton systems can be found in many places. ${ }^{48}$ These classical equations of motion with a quantum-mechanically computed susceptibility can be directly quantized. The effective quantum Hamiltonian follows from the resulting quantum equations of motion.

In addition to absorption, two-photon emission which provides a two-photon lasing mechanism is also described by (5.1). In considering the conditions for stimulated two-photon emission in either the standing-wave or traveling-wave configuration with loss included, it is easily found that stimulated emission cannot be set up from spontaneous emission alone. A sufficiently strong field at the lasing frequency $\omega$ has to be present initially. ${ }^{49-51}$ Therefore we consider the two-photon lasing configurations depicted in Fig. 2. It is not the purpose of this article to present the semiclassical theory of two-photon laser operation, or to discuss the operating consideration in a realistic material system. But the following facts may be observed:

In the traveling-wave configuration, Fig. 2(a), the gain is obtained from a large external pump field which may have many frequencies but which is still in a single temporal mode. Neglecting various loss mechanisms, this lasing process can be described by the interaction Hamiltonian (5.1) with an initial radiation state $\rho_{0}$ having a nonzero amount of power, $\operatorname{tr} \rho_{0} a^{\dagger} a \neq 0$. If a laser is used for the external pump, $\rho_{0}=|\alpha\rangle\langle\alpha|$, but intense thermal light can also be used. The radiation state $\rho$, as well as other dynamical variables, are spatially dependent in this case. In the standing-wave case, Fig. 2(b), only a single standing-wave mode is under consideration. The gain is initially obtained from the usual one-photon lasing mechanism, the host atoms for both lasing transitions being contained in the same cavity. ${ }^{49}$ The Hamiltonian is now

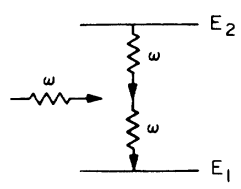

(a) $E_{2}-E_{1}=2 \hbar_{\omega}$

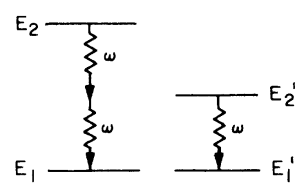

(b) $E_{2}-E_{1}=2 \hbar_{\omega}$ $E_{2}^{\prime}-E_{1}^{\prime}=\hbar_{\omega}$
FIG. 2. Two possible configurations for stimulated two-photon emission. 


$$
H=\hbar\left(\omega a^{\dagger} a+p_{2} M_{2} a^{\dagger 2}+p_{2}^{*} M_{2}^{\dagger} a^{2}+p_{1} M_{1} a^{\dagger}+p_{1}^{*} M_{1}^{\dagger} a\right),
$$

with a vacuum initial state; the subscripts 2 and 1 for $p$ and $M$ refer to the two-photon and one-photon lasing material. Population inversion is required in both configurations. Other possible configurations for two-photon lasers can be developed, but these two seem to be the most important.

Spontaneous two-photon emission has been experimentally observed, ${ }^{52}$ but not stimulated twophoton emission. Two-photon stimulated emission was first suggested for giant pulse generation. ${ }^{49}$

When such a two-photon laser operates far above threshold with amplitude and phase stability, the fluctuation in $M$ should be relatively small, so that $M$ is approximately a $c$ number. By ignoring the quantum nature of $M,(5.2)$ or $(5.3)$ becomes an $H_{Q}$ of the form (4.2). If the dissipative mechanisms are also neglected, (4.12) shows that a TSC $|\beta\rangle_{g}$ will be obtained. A similar argument was used by Glauber $^{18}$ to suggest that a coherent state describes one-photon laser radiation far above threshold. As we show in the Appendix, this can be formalized in the so-called self-consistent-field approximation (SCFA), which has been used in one-photon laser theory..$^{53,54}$ Therefore a two-photon device of this type operating well above threshold can be expected to produce a state $|\beta\rangle_{g}$ with further small classically random fluctuations in the variable $\beta$, in a way exactly analogous to one-photon lasers where $|\alpha\rangle$ is produced with small classical fluctuations in $\alpha$. This argument can be made for a standingwave, a traveling-wave, or an arbitrary spatialtemporal mode.

\section{B. Effect of perturbations in the two-photon system}

An additive Gaussian noise and a damping mechanism can be introduced to account for some unavoidable sources of noise similar to the one-photon case, while still preserving that distinguishing feature of TCS's with $\left\langle\Delta a_{1}^{\prime 2}\right\rangle$ and $\left\langle\Delta a_{2}^{\prime 2}\right\rangle$ smaller than $\frac{1}{4}$. This can be illustrated as follows: The quantum effect of loss is incorporated through the density operator relaxation equation

$$
\begin{aligned}
\frac{\partial \rho}{\partial t}= & -\frac{i}{h}\left[H_{Q}, \rho\right]+\frac{1}{2} \gamma\left(2 a \rho a^{\dagger}-a^{\dagger} a \rho-\rho a^{\dagger} a\right) \\
& +\gamma \bar{n}\left(a^{\dagger} \rho a+a \rho a^{\dagger}-a^{\dagger} a \rho-\rho a a^{\dagger}\right),
\end{aligned}
$$

with $H_{Q}$ given by $(4.2)$. The damping rate is $\gamma$ and $\bar{n}$ represents the thermal noise contribution from the reservoir that couples to the radiation field. This quantum description of loss has been extensively discussed in the literature. ${ }^{55-57}$ For an initial coherent state, (5.3) can be solved exactly through the Fokker-Planck equations for $\rho^{(n)}\left(\alpha^{*}, \alpha\right)$. In particular, all of the characteristic functions are still in Gaussian form, ${ }^{58}$ and for $\gamma \neq 4 r$,

$$
\begin{aligned}
\left\langle\Delta a_{1,2}^{\prime 2}(t)\right\rangle= & \frac{1}{4} \frac{\gamma}{\gamma \pm 4 r} \pm \frac{r}{\gamma \pm 4 r} e^{-(\gamma \pm 4 r) t} \\
& +\frac{\gamma \bar{n}}{2(\gamma \pm 4 r)}\left(1-e^{-(\gamma \pm 4 r) t}\right),
\end{aligned}
$$

with $a^{\prime}=a e^{i \omega t}$. It can be readily shown that $\left\langle\Delta a_{j}^{\prime 2}(t)\right\rangle$ $>0$ and that the uncertainty principle is obeyed for all $t$. Note that even when $\bar{n}=0$, the state is no longer pure in the presence of $\gamma$. Asymptotically we have

$$
\left\langle\Delta a_{1}^{\prime 2}(t)\right\rangle \rightarrow \gamma \bar{n} / 8 r,\left\langle\Delta a_{2}^{\prime 2}(t)\right\rangle \rightarrow \infty ; t \rightarrow \infty, 2 r \gg \gamma .
$$

The condition $2 r \gg \gamma$ can be satisfied together with the stability condition (4.3) for the usual values of $\gamma$

$$
\omega>2 r \gg \gamma \text {. }
$$

Thus for $\bar{n} \lesssim 1$ the corresponding state approaches an eigenstate of $a_{1}$. In any case the quantum fluctuation in $a_{1}$ vanishes as $t \rightarrow \infty$.

From the results of Sec. IV B, it can be seen that two-photon laser is a phase-sensitive device. If a uniform random phase is assumed, i.e., if $a^{\prime}=a e^{j \phi}$ for $\phi$ uniformly distributed in $[-\pi, \pi]$ is the dynamical observable one has to deal with, then

$$
\left\langle\Delta a_{1}^{\prime 2}\right\rangle=\left\langle\Delta a_{2}^{\prime 2}\right\rangle=\frac{1}{2}\left(\left\langle\Delta a_{1}^{2}\right\rangle+\left\langle\Delta a_{2}^{2}\right\rangle\right) .
$$

Equation (5.7) contributes a noise greater than $\frac{1}{4}$ for both quadrature components and one distinguishing characteristic of a TCS is lost. The detailed theories of one-photon lasers show that amplitude fluctuation is suppressed in a laser operating far above threshold, and the phase fluctuation is also inversely proportional to the average photon numbers. Moreover, these amplitude stabilization and phase stabilization properties are general features of a wide class of self-sustained oscillators, quantum or classical. 59,55 If the twophoton laser belongs to this class, the phase would diffuse away from some initially measured value only slowly for high-power operation similar to the one-photon case. The phase can then be tracked and the relative phase determined accurately. As a result the field may be regarded to be in a true TCS. Whether the perturbation introduced by the atomic fluctuations may indeed be sufficiently small so that $|\beta\rangle_{g}$ does not become $|\beta\rangle$ can be determined only by explicitly working out the detailed quantum theory of two-photon lasers. The quantum theory of one-photon lasers has been extensively developed, ${ }^{55-57}$ but the two-photon laser requires more careful approximations because of the small 
noise in one quadrature component and the more delicate equations of motion involved. Hopefully, the TCS picture can be checked experimentally, as discussed in Sec. VI, by practically realizing a two-photon laser. In any case, $|\beta\rangle_{g}$ serves as the basic description to be averaged further by a classical density function in the presence of other random interference.

Recently, the quantum theory of multiphoton lasers, including a quantum treatment of the atoms and the loss mechanism, was discussed by McNeil and Walls..$^{59-61}$ However, the nature of their approximations was not clarified and they produced lasing results from models ${ }^{59,60}$ which cannot lase because of the absence of an input field to provide a nonzero gain. While some qualitative features of their two-photon results are in agreement with the TCS picture, such as large total fluctuation from (3.35), they suggest that the photon statistics are similar to the one-photon case. The Poissonlike distribution they find in Ref. 59 is obtained for an unrealistic loss model, in addition to the above error. The lossless photon distribution they obtain in Ref. 61 is neither Poisson nor of the form (3.23). Since the atomic populations rather than the atomic polarizations were treated as a constant in the derivation of that distribution, it appears that it is valid near, instead of far above, threshold. A complete and careful calculation of the two-photon laser state remains to be carried out.

\section{Degenerate parametric amplifier}

A degenerate parametric amplifier interpretation can be given to (4.2) similar to the above two-photon laser interpretation. When the material system is suppressed through a susceptibility and when the pump field is treated classically, a twophoton coherent state $|\beta\rangle_{g}$ would be obtained from a degenerate parametric amplifier such as the one depicted in Fig. 3. An intense initial field at frequency $\omega$ has to be present in addition to the pump field for degenerate parametric amplification. A TCS is already present in the treatment of nondegenerate parametric amplifiers by Mollow and Glauber $^{47}$ in the joint Wigner distribution for the signal and idler modes, even though a coherent state plus noise is obtained for each of these modes. ${ }^{62}$ That a TCS can be generated in a singlemode degenerate parametric amplifier was also observed by Stoler ${ }^{11}$ and by Lu. ${ }^{10}$

The phase-sensitive behavior of a two-photon laser is reminiscent of parametric processes, and a degenerate parametric amplifier is a kind of twophoton laser. A basic distinction, however, can be made. In the parametric case the final and in- itial states of the atoms are identical, but they are different in the two-photon case. The question of quantum statistics is similar in both cases. The pump field of Fig. 3 should be treated as a quantum degree of freedom. A fully quantum treatment of degenerate parametric oscillators can be equally or more complex than a fully quantum treatment of two-photon lasers, depending on whether the atomic variables are included in the parametric case and whether the important intermediate state is included in the two-photon case when present.

No single-mode degenerate parametric oscillation above threshold has been observed. Careful consideration has to be given to any possible competing processes in the actual development of a two-photon laser or degenerate parametric amplifier, such as third-harmonic generation.

\section{COHERENCE AND PHOTON COUNTING}

Two-photon coherent states are not coherent according to Glauber's original definition. ${ }^{63}$ That is, they do not factorize the correlation functions which describe photon counting experiments employing ordinary photodetectors. However, they factorize a different set of correlation functions obtained by transforming $a$ to $b$ in the field expansion. This kind of transformed correlation function gives the joint counting rates for detectors operating with an interaction mechanism different from the usual dipole absorption, ${ }^{18}$ in analogy with detectors that function by stimulated emission discussed by Mandel. ${ }^{64}$ Thus a TCS is fully coherent in a sense closely related to the usual definition of optical coherence. Further detailed discussion can be given only in the multimode treatment of TCS.

Here let us consider photon counting experiments associated with a single radiation mode whose spatial dependence has been integrated through the spatial response of a photoabsorption detector. For any state $\rho$ the distribution $\langle n|\rho| n\rangle$ can be generated by an ideal detector if the counting time $T$ is large enough. ${ }^{65}$ Therefore we consider the generating function

$$
\begin{aligned}
& Q(\lambda)=\sum_{n=0}^{\infty}(1-\lambda)^{n}\langle n|\rho| n\rangle, \\
&\left\langle a^{\dagger n} a^{n}\right\rangle=\langle N \cdots(N-1) \cdots(N-n+1)\rangle \\
&=\left.(-1)^{n} \frac{\partial^{n} Q}{\partial \lambda^{n}}\right|_{\lambda=0} \cdot
\end{aligned}
$$

With the Glauber model ${ }^{18}$ of an $n_{0}$-atom photodetector with quantum efficiency $s$ and a sufficiently small counting time $T$, the generating function for the counting distribution $p(n, T)$ of registering $n$ 
counts in time $T$ is merely a scaling of (6.1),

$$
p(n, T)=\left.\frac{(-1)^{n}}{n !} \frac{\partial^{n} Q\left(s n_{0} T \lambda\right)}{\partial \lambda^{n}}\right|_{\lambda=1} .
$$

From (3.23) the function $Q(\lambda)$ for a TCS can be evaluated in closed form using the summation formula for Hermite polynomials, ${ }^{33}$

$$
\begin{gathered}
Q(\lambda)=\tau^{1 / 2} \exp \left[[(1-\lambda) \tau-1]|\beta|^{2}\right. \\
\left.+\left[1-(1-\lambda)^{2} \tau\right]\left(\frac{\nu^{*}}{2 \mu} \beta^{2}+\frac{\nu}{2 \mu^{*}} \beta^{* 2}\right)\right], \\
\tau \equiv\left[|\mu|^{2}-(1-\lambda)^{2}|\nu|^{2}\right]^{-1} .
\end{gathered}
$$

From (6.5) the coefficients $g_{n}=\left\langle a^{\dagger n} a^{n}\right\rangle /\left\langle a^{\dagger} a\right\rangle^{n}$ for a first-order coherent field ${ }^{18}$ can be computed by (6.3). In particular, $\left\langle a^{\dagger} a\right\rangle=|\nu|^{2}+|\hat{\beta}|^{2}$, as given by (3.29), and

$$
\begin{aligned}
\left\langle a^{\dagger 2} a^{2}\right\rangle= & \left\langle a^{\dagger} a\right\rangle^{2}+2|\beta|^{2}\left(4|\nu|^{2}+3\right)|\nu|^{2} \\
& -\left(\mu^{*} \nu^{*} \beta^{2}+\mu \nu \beta^{* 2}\right)\left(1+4|\nu|^{2}\right)+|\nu|^{2}+2|\nu|^{4} .
\end{aligned}
$$

Any value $g_{2}>0$ can be generated from (6.6) by proper choice of $(\mu, \nu, \beta)$. In particular, $g_{2}<1$ for a TCS if and only if

$$
\begin{aligned}
2|\nu|^{4}+|\nu|^{2}+2 & \left(4|\nu|^{2}+3\right)|\nu|^{2}|\beta|^{2} \\
& <\left(\mu^{*} \nu^{*} \beta^{2}+\mu \nu \beta^{* 2}\right)\left(1+4|\nu|^{2}\right),
\end{aligned}
$$

and $g_{2} \geq 1$ if the sign is reversed. For $\beta=0$, we always have $g_{2}>1$ from the noise $|\nu|^{2}$, but $g_{2}<1$ is clearly possible for small $\nu$ and large $\mu$ with nonvanishing $\beta$. Recall that $g_{2}<1$ is a characteristic quantum effect without classical analog, and that currently available sources all generate $g_{2} \geq 1$. Stoler ${ }^{11}$ and $\mathrm{Lu}^{10}$ have previously observed that $g_{2}$ $<1$ may be obtained from a state of this kind.

For most values of $(\mu, \nu), g_{2}$ can be tuned by varying $\beta$ in a TCS from (6.7), exhibiting both anticorrelation $\left(g_{2}<1\right)$ and enhanced correlation $\left(g_{2}\right.$ $>2$ ) effects. This fact permits one to check whether a certain particular TCS is obtained by a HanburyBrown-Twiss experiment. The photon counting distribution (3.23) or (6.4) is also sufficiently different froma Poisson distribution that its measurement will reveal the presence of a TCS. The in-

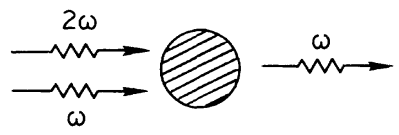

FIG. 3. Phase-matched degenerate parametric amplification with pump field at frequency $2 \omega$. fluence of nonideal detectors and noise on these photocount statistics should be worked out in detail for actual comparison with experiments.

\section{APPLICATIONS OF TWO-PHOTON COHERENT STATES}

We will briefly describe some novel applications of two-photon lasing processes which result from the quantum properties of TCS. Quite apart from their quantum characteristics, two-photon lasers also exhibit certain pulse-shortening behavior absent in the one-photon case..$^{51}$ Their particular nonlinearity may lead to many interesting phenomena and applications, including the realization of very-high-power lasers. However, only certain applications of TCS's will be treated here. These applications also illustrate the essential differences between TCS's and ordinary coherent states.

\section{A. Ideal linear amplifier}

Under the action of a two-photon amplifier, an input coherent state $|\beta\rangle$ will be changed to $|\beta\rangle_{g}$, from (4.45). For simplicity we assume $\mu$ and $\nu$ to be real. From (3.28),

$$
\begin{aligned}
& \left\langle a_{1}\right\rangle_{g}=\hat{\beta_{1}}=(\mu-\nu) \beta_{1}, \\
& \left\langle a_{2}\right\rangle_{g}=\hat{\beta_{2}}=(\mu+\nu) \beta_{2} .
\end{aligned}
$$

Thus one of the original quadrature components is linearly amplified while the other is linearly attenuated. The nonlinearity of a two-photon amplifier is therefore of the simplest kind; it is piecewise linear.

To be definite let $\nu>0$. The quantum noise $\left\langle\Delta \beta_{1}^{2}\right\rangle$ decreases from $\left\langle\Delta \beta_{1}^{2}\right\rangle=\frac{1}{4}$ to $\left\langle\Delta \hat{\beta}_{1}^{2}\right\rangle=\frac{1}{4}(\mu-\nu)^{2}$, while that of $\hat{\beta}_{2}$ increases to $\frac{1}{4}(\mu+\nu)^{2}$, from (3.31). If we define the signal-to-noise ratio

$$
(S / N)_{j \rho}=\left\langle a_{j}\right\rangle^{2} /\left\langle\Delta a_{j}^{2}\right\rangle, \quad j=1,2,
$$

where the average is taken in a state $\rho$, we find that $(S / N)_{j}$ is invariant in the amplification and attenuation, i.e.,

$$
(S / N)_{j|\beta\rangle}=(S / N)_{j|\beta\rangle_{g}}
$$

The above definition of signal-to-noise ratio is meaningful. If we try to determine the value of $\beta_{2}$ by measuring $a_{2}$ in a coherent state or TCS, (7.2) provides a standard or measure on how well one may estimate $\beta_{2},{ }^{66,1}$ or equivalently how "noisy" $\beta_{2}$ is in the "best" measurement.

We can interpret $|\beta\rangle$ as the state of an optical signal presented to a receiver which generates $|\beta\rangle_{g}$ by a two-photon amplifier $U_{Q}\left(t, t_{0}\right)$ and then measures $a_{2}$ on $|\beta\rangle_{g}$. It is obvious physically that the signal-to-noise ratio cannot be improved by amplification. The introduction of $f_{3}$ into $U_{Q}$ does 
not increase the signal energy, i.e., the part of which is proportional to $\beta_{2}^{2}$. The advantage of amplification is practical, similar to the classical case. Raising the signal level makes possible easier measurement of $\beta_{2}$. Since the two-photon amplification process (4.45) operates above threshold, it can be superior to one-photon amplifiers operating below threshold in its possibilities of yielding higher gain and lower noise. If amplification for both quadrature components is desired, the input beam can be first split and two different $U_{Q}\left(t, t_{0}\right)$ applied to $\beta_{1}$ and $\beta_{2}$ separately. Ideally, no signal-to-noise degradation will occur from (7.3).

The above amplifier performance is not sensitive to the addition of a Gaussian noise. Since we are now utilizing the bigger noise component, there is no need to maintain $\left\langle\Delta a_{1}^{2}\right\rangle<\frac{1}{4}$ and a random phase would not seriously degrade the system performance. Therefore the process described by $U_{Q}\left(t, t_{0}\right)$ provides a possible mechanism in the realization of an ideal linear amplifier.

\section{B. Local oscillator}

One may take advantage of the absolutely small $\left\langle\Delta a_{1}^{2}\right\rangle \ll \frac{1}{4}$ in $|\beta\rangle_{g}$ in the reception of a coherentstate signal with a size-limited detector. By employing a local oscillator which generates radiation in state $|\beta\rangle_{g}$, it is possible to attenuate the quantum noise by an amount which compensates the large diffraction loss incurred in free space or unguided propagation. This is achieved by coupling a mode in $|\beta\rangle_{g}$ to the incoming mode in $|\alpha\rangle$ in the form of a new mode

$$
a_{R}=\epsilon a_{S}+\left(1-\epsilon^{2}\right)^{1 / 2} a_{L},
$$

where $\epsilon^{2}$ represents the fraction of energy received and $a_{S}, a_{L}$, and $a_{R}$ are the photon annihilation operators for the signal, the local oscillator, and the receiver mode. The quantum noise in $a_{R}$ is clearly dominated by that of $a_{L}$ for small $\epsilon^{2}$. The signal-to-noise ratio in $a_{R_{1}}$ becomes $\sim \frac{1}{4} \alpha_{1}^{2}$ for $\left\langle\Delta a_{L 1}^{2}\right\rangle \simeq 0$, whereas it is $\frac{1}{4} \epsilon^{2} \alpha_{1}^{2}$ for a coherentstate local mode $\left\langle\Delta a_{L 1}^{2}\right\rangle=\frac{1}{4}$. Further discussion can be found in Ref. 1. Note that this advantage of $\left\langle\Delta a_{L 1}^{2}\right\rangle \ll \frac{1}{4}$ cannot be obtained if a one-photon-laser local oscillator is used to produce a coherentstate receiver mode $a_{R}$ and a $U_{Q}\left(t, t_{0}\right)$ subsequently applied, because of the corresponding signal attenuation. An absolutely small $\left\langle\Delta a_{L 1}^{2}\right\rangle$ is needed in this scheme.

If an arbitrary amount of power is available, it is possible to have a state with $\left\langle\Delta a_{1}^{2}\right\rangle \rightarrow 0$. However, for a fixed total radiation energy

$$
\operatorname{tr} \rho a^{\dagger} a \leq S,
$$

a decrease of $\left\langle\Delta a_{1}^{2}\right\rangle$ in $|\beta\rangle_{g}$ can be obtained only at the expense of spending a portion of available energy $S$ in the form of added quantum noise energy $|\nu|^{2}$, from (3.35). Thus $\left\langle\Delta a_{1}^{2}\right\rangle \rightarrow 0$ requires $S \rightarrow \infty$. This consideration is not important in the context of local oscillators at a receiver where large enough amount of power is usually available to make $\left\langle\Delta a_{1}^{2}\right\rangle$ sufficiently small. A four-orders-ofmagnitude reduction from the quantum noise $\frac{1}{4}$ of $\alpha_{1}\left(|\nu|^{2} \simeq 10^{2}\right)$ for a GHz bandwidth optical signal at $\omega / 2 \pi \simeq 10^{15} \mathrm{~Hz}$ requires only a $|\nu|^{2}$ corresponding to $\sim 1 \mu \mathrm{W}$. This will usually bring it down to the level of other extraneous noises. Furthermore, even a ten-orders-of-magnitude reduction of the quantum noise requires $\sim 1 \mathrm{~W}$ under the same condition.

Other practical considerations also should not invalidate the utilization of this small-noise property. With proper signaling and reception schemes one can always observe the signal quadrature component without significant degradation from random-phase modulation. From (4.43) it is assured that free-space propagation of $|\beta\rangle_{g}$ will not disturb the quantum noise characteristics. The rotation (3.59) can be readily compensated in an optical receiver without affecting the signal level, so that TCS's are as good as minimum-uncertainty states in this application. These and many other problems involved in a realistic communication system will be treated elsewhere.

\section{Transmission source}

Further performance improvement can be obtained if the transmitter generates informationcarrying radiation in a $\mathrm{TCS}$. In this case the radiation power constraint (7.5) poses a major limitation on the achievable signal-to-noise ratio. It has been shown that ${ }^{67}$ TCS's provide the maximum $(S / N)_{1}$ obtainable for one of the quadrature components among all possible states satisfying (7.5). By spending a fraction $S /(2 S+1)$ of $S$ as quantum noise energy with, e.g.,

$$
\begin{aligned}
& \mu=(S+1) /(2 S+1)^{-1 / 2}, \quad \nu=S /(2 S+1)^{-1 / 2}, \\
& \beta_{2}=\hat{\beta}_{2}=0,
\end{aligned}
$$

we have

$$
(S / N)_{1|\beta\rangle_{g}}=4\left(S^{2}+S\right) .
$$

Compared to the signal-to-noise ratio

$$
(S / N)_{1|\beta\rangle}=4 S
$$

in a coherent state, we see that the available "signal energy" is effectively increased from $S$ to $S^{2}$ $+S$. This is actually a reduction of quantum noise.$^{68}$ Among other things, this increase in $(S / N)_{1}$ leads to a higher information capacity even when the 
other quadrature component is not employed for information transmission. ${ }^{1}$ In the presence of other extraneous noise, quantum noise reduction in a transmitter state $|\beta\rangle_{g}$ can also be obtained with only a relatively small $|\nu|^{2}$, as illustrated above.

Finally, we observe that the new photocount statistics may itself lead to novel applications in communications.

\section{CONCLUDING REMARKS}

We have discussed in some detail the properties of a class of radiation states which are closely related to the well-known coherent states. These two-photon coherent states possess a number of distinguishing characteristics which lead to potential device applications for quantum noise reduction. They may also find application in experimental situations that require low noise sensitivity. It has also been suggested that these states can be obtained from two-photon stimulated processes, or equivalently that they provide a good description of two-photon laser states. The ultimate validity of this proposition must be tested by an elaborated quantum-statistical theory of two-photon lasing processes and above all, hopefully, by experiment.

Our present work was motivated by the desire to realize physically certain specific quantum states and quantum measurements for practical applications. It appears that the general problems of state and measurement synthesis are interesting and useful areas in quantum optics and electronics, as well as in the foundations of quantum mechanics.

\section{ACKNOWLEDGMENT}

The author thanks the following persons for heipful discussions relating to various parts of the pa- per: R. S. Kennedy, M. Lax, J. H. Shapiro, H. S. Tye, and S. Y. Yuen.

\section{APPENDIX: TWO-PHOTON LASING STATE IN THE SCFA}

Here it is shown that the radiation state of a twophoton laser is a TCS in the self-consistent-field approximation (SCFA), ${ }^{53,54}$ if loss is also neglected. Let $H$ be the total Hamiltonian of an atom-field system:

$$
H=H_{\text {atom }}+H_{\text {field }}+H_{\text {int }},
$$

with $H_{\text {field }}+H_{\text {int }}$ given by (5.2). In the SCFA, the total density operator is assumed to factorize,

$$
\rho=\rho_{a t} \otimes \rho_{f d} \text {. }
$$

The equation of motion for the radiation density operator $\rho_{f d}$ can be obtained from that of $\rho$ by tracing over the atomic variables with the result

$$
\frac{\partial \rho_{f d}}{\partial t}=-\frac{i}{\hbar}\left[H_{Q}, \rho_{f d}\right]
$$

where $H_{Q}$ is given by $(4.2)$, with

$$
f_{1}=\omega, f_{2}=p_{2} \operatorname{tr}\left(M_{2} \rho_{a t}\right), f_{3}=p_{1} \operatorname{tr}\left(M_{1} \rho_{a t}\right) .
$$

Thus a TCS will be developed from an initial coherent state from (4.12), and (A4) gives the effective field Hamiltonian under the SCFA. Note that a resonant factor $e^{-2 i \omega t}$ is automatically present in $f_{2}$, from the atomic resonance ir $M$.

Even though the above derivation is given for a single frequency, it applies equally to any spatialtemporal mode if $H_{I}$ is in the form (5.1). The same result is obtained for a parametric oscillator by interpreting $M_{1}$ as the pump photon destruction operator and $p_{1}$ as the susceptibility, with $M_{2}=0$. However, it seems less justified to neglect the quantum fluctuation of the pump photon, which is also described by a boson operator as the signal mode.
*Work supported by the National Aeronautics and Space Administration under Grant No. NGL 22-009-013 and by the Office of Naval Research under Contract No. NR042-342.

${ }^{1} \mathrm{H}$. P. Yuen, in Proceedings of the 1975 Conference on Information Sciences and Systems (Johns Hopkins U.P., Baltimore, 1975), p. 171.

${ }^{2}$ Coherent states are excluded when we refer to a minimum-uncertainty state. For a mode of frequency $\omega$, coherent states are minimum-uncertainty states with $u^{2}\left\langle\Delta q^{2}\right\rangle=\left\langle\Delta p^{2}\right\rangle=\frac{1}{2} \hbar \omega$. Minimum-uncertainty wave packets are treated in almost every textbook in quantum mechanics; see also Ref. 3. The distinction between minimum-uncertainty radiation states and coherent states is clarified in Sec. III E.

${ }^{3}$ W. H. Louisell, Quantum Statistical Properties of Radiation (Wiley, New York, 1973).

${ }^{4}$ R. J. Glauber, Phys. Rev. 131, 2766 (1963); Phys. Rev.
Lett. 10, 84 (1963).

${ }^{5}$ It is actually a power spectral density, i.e., power per unit frequency.

${ }^{6} \mathrm{H}$. P. Yuen, Phys. Lett. 51A, 1 (1975).

${ }^{7} \mathrm{~N}$. Bloembergen, Nonlinear Optics (Benjamin, New York, 1965).

${ }^{8}$ E. H. Kennard, Z. Phys. 44, 326 (1927).

${ }^{9}$ D. Stoler, Phys. Rev. D $\underline{1}, 3217$ (1970); 4 , 1925 (1971).

${ }^{10}$ E. Y. C. Lu, Nuovo Cimento Lett. $\underline{2}, 1241$ (1971); $\underline{3}$, 585 (1972).

${ }^{11}$ D. Stoler, Phys. Rev. Lett. 23 , 1397 (1974).

${ }^{12}$ B. R. Mollow, Phys. Rev. $\underline{162}, 1256$ (1967).

${ }^{13}$ J. Perina, V. Perinova, and R. Horak, Czech. J. Phys. B 23, 975 (1973); J. Perina, V. Perinova, and L. Mista ibid. 24, 482 (1974).

${ }^{14} \mathrm{D}$. W. Robinson, Commun. Math. Phys. 1, 159 (1965).

${ }^{15} \mathrm{~J}$. Manuceau and A. Verbeure, Commun. Math. Phys. 9, 293 (1968). 
${ }^{16} \mathrm{~F}$. A. Berezin, The Method of Second Quantization (Academic, New York, 1966).

${ }^{17} \mathrm{R}$. Haag, in Lectures in Theoretical Physics, edited by W. E. Brittin and A. O. Barut (University of Colorado Press, Denver, 1960), Vol. XX, p. 353.

${ }^{18} \mathrm{R}$. J. Glauber, in Quantum Optics and Electronics, edited by C. M. DeWitt et al. (Gordon and Breach, New York, 1965), p. 63

${ }^{19}$ J. R. Klauder and E. C. G. Sudarshan, Fundamentals of Quantum Optics (Benjamin, New York, 1968).

${ }^{20} \mathrm{~V}$. Bargmann, Commun. Pure Appl. Math. 14, 187 (1961).

${ }^{21}$ C. L. Mehta and E. C. G. Sudarshan, Phys. Rev. 138, B274 (1965).

${ }^{22}$ J. R. Klauder, J. McKenna, and D. G. Currie, J. Math. Phys. 6, 733 (1965).

${ }^{23}$ J. R. Klauder, Phys. Rev. Lett. 16, 534 (1966).

${ }^{24}$ M. M. Miller and E. A. Mishkin, Phys. Rev. 164, 1610 (1967).

${ }^{25}$ E. C. G. Sudarshan, Phys. Rev. Lett. 10, 277 (1963).

${ }^{26} \mathrm{R}$. J. Glauber, in Physics of Quantum Electronics, edited by P. L. Kelly et al. (McGraw-Hill, New York, 1966), p. 788.

${ }^{27} \mathrm{R}$. J. Glauber, in Laser Handbook, edited by F. T. Arecchi and E. O. Schulz-DuBois (Elsevier, New York, 1972), Vol. I, p. 1.

${ }^{28}$ The term "probability distribution" is used instead by many physicists. In the mathematics and engineering literature, the integral of a density function is called a probability distribution function.

${ }^{29}$ E. Arthurs and J. L. Kelly, Jr., Bell Syst. Tech. J. 44, 725 (1965).

${ }^{30} \mathrm{~J}$. P. Gordon and W. H. Louisell, in Physics of Quantum Electronics, edited by P. L. Kelley et al . (McGrawHill, New York, 1966), p. 833.

${ }^{31}$ H. P. Yuen and M. Lax, IEEE Trans. Inf. Theory IT-19, 740 (1973).

${ }^{32} \mathrm{~J}$. von Neumann, Math. Ann. 104, 570 (1931).

${ }^{33}$ N. N. Lebedev, Special Functions and their Applications (Prentice-Hall, Englewood Cliffs, 1965).

${ }^{34}$ States with $\Lambda \geq 0$ (positive semidefinite), i.e., with one eigenvalue of $\Lambda$ equal to zero, are the limiting case of the normalizable TCS, such as the eigenstates of $a_{1}$ or $a_{2}$. They are not normalizable and have an infinite average photon number from (3.35). Strictly speaking, these states are outside the Hilbert space of states.

${ }^{35} \mathrm{R}$. Bellman, Introduction to Matrix Analysis (McGrawHill, New York, 1970), p. 112.

${ }^{36}$ Two-photon coherent states were originally called "generalized coherent states" (GCS) in Ref. 1 and 6 by this author. I have since learned that GCS has been used to denote the general states introduced by $\mathrm{U}$. M. Titulaer and R. J. Glauber [Phys. Rev. 145, 1041 (1966)] which satisfy the full coherence conditions of Glauber (Ref. 18). See, e.g., J. Perina, Coherence of Light (Van Nostrand, New York, 1972), p. 186.

${ }^{37}$ N. H. McCoy, Proc. Edinb. Math. Soc. 3, 118 (1932).

${ }^{38}$ Such a positive definite spectrum condition is a basic postulate of quantum field theory; see, e.g., R. Haag and B. Schrör, J. Math. Phys. $\underline{3}, 248$ (1962).
${ }^{39}$ N. N. Bogoliubov, J. Phys. USSR 11, 23 (1947).

${ }^{40} \mathrm{H}$. T. Davis, Introduction to Nonlinear Differential and Integral Equations (Dover, New York, 1962).

${ }^{41}$ W. T. Reid, Riccati Differential Equations (Academic, New York, 1972).

${ }^{42} \mathrm{R}$. Guccione and J. Van Kranendonk, Phys. Rev. Lett. 14, 583 (1965).

${ }^{43} \mathrm{M}$. Iannuzzi and E. Polacco, Phys. Rev. Lett. 13, 371 (1964).

${ }^{44}$ R. Guccione-Gush, H. P. Gush, and J. van Kranendonk, Can. J. Phys. 45, 2513 (1967).

${ }^{45} \mathrm{D}$. Prichard, J. Apt. and T. W. Ducas, Phys. Rev. Lett. 32, 641 (1974); M. D. Levenson and N. Bloembergen, Phys. Rev. Lett. 32, 645 (1974); P. F. Liao and J. E. Bjorkholm, Phys. Rev. Lett. 34, 1 (1975).

${ }^{46}$ W. H. Louisell, A. Yariv, and A. E. Siegman, Phys. Rev. 124, 1646 (1961).

${ }^{47}$ B. R. Mollow and R. J. Glauber, Phys. Rev. 160, 1076 (1967); 160, 1097 (1967).

${ }^{48}$ See, e.g., E. M. Belenov and I. A. Poluektov, Zh. Eksp. Teor. Fiz. 56, 1407 (1969) [Sov. Phys.—JETP 29, 754 (1969)] ; L. E. Estes, L. M. Narducci, and B. Shammas, Nuovo Cimento Lett. 1, 775 (1971); M. Takatsuji, Phys. Rev. A 4, 808 (1971).

${ }^{49}$ P. P. Sorokin and N. Braslau, IBM J. Res. Dev. $\underline{8}, 117$ (1964).

${ }^{50}$ V. S. Letokhov, Zh. Eksp. Teor. Fiz. Pis'ma Red. 1 , 284 (1968) [JETP Lett. 7, 221 (1968)] .

${ }^{51}$ H. P. Yuen, Appl. Phys. Lett. 26, 505 (1975).

${ }^{52} \mathrm{M}$. Lipeles, R. Novick, and N. Tolk, Phys. Rev. Lett. 15,690 (1965).

${ }^{53}$ C. R. Willis, J. Math. Phys. 5, 1241 (1964).

${ }^{54}$ W. Weidlich and F. Haake, Z. Phys. 186, 203 (1965).

${ }^{55}$ M. Lax, in Statistical Physics, edited by M. Chretien et al. (Gordon and Breach, New York, 1968), Vol. II, p. 271.

${ }^{56} \mathrm{H}$. Haken, in Encyclopedia of Physics (Springer, New York, 1970), Vol. XXV/2C.

${ }^{57}$ M. O. Scully and W. E. Lamb, Jr., Phys. Rev. 159, 208 (1967); M. Sargent III, M. O. Scully, and W. E. Lamb, Jr., Laser Physics (Addison Wesley, Reading, Mass., 1974).

${ }^{58}$ The exact solution will be derived and discussed for the general multimode case in a separate treatment.

${ }^{59}$ K. J. McNeil and D. F. Walls, J. Phys. A 8,104 (1975).

${ }^{60} \mathrm{~K}$. J. McNeil and D. F. Walls, J. Phys. A $\overline{8}, 111$ (1975).

${ }^{61}$ K. J. McNeil and D. F. Walls, Phys. Lett. $51 \mathrm{~A}, 233$ (1975).

${ }^{62} \mathrm{~A}$ proper mixing of the signal and idler modes will produce a TCS.

${ }^{63}$ R. J. Glauber, Phys. Rev. 130, 2529 (1963).

${ }^{64}$ L. Mandel, Phys. Rev. 152, 438 (1966).

${ }^{65}$ See, e.g., Sec. 8-3 of Ref. 19.

${ }^{66}$ S. D. Personick, IEEE Trans. Inf. Theory IT-17, 240 (1971).

${ }^{67}$ H. P. Yuen, Phys. Lett. 56A, 105 (1976).

${ }^{68}$ There is no contradiction between (7.8) and (7.9) and (7.3), because $U_{Q} D(\beta)|0\rangle$ is not the most efficient way of generating $|\beta\rangle_{\mathrm{g}}$ in terms of the constraint (7.5). 